\title{
Stochastic Hysteresis and Resonance in a Kinetic Ising System
}

\author{
S. W. Sides, ${ }^{a * \dagger \ddagger}$ P. A. Rikvold, ${ }^{\mathrm{b} * \dagger \ddagger}$ and M. A. Novotny ${ }^{\mathrm{c} \dagger}$ \\ ${ }^{*}$ Center for Materials Research and Technology and Department of Physics, and \\ ${ }^{\dagger}$ Supercomputer Computations Research Institute, \\ Florida State University, Tallahassee, Florida 32306-4130 \\ ${ }^{\ddagger}$ Colorado Center for Chaos and Complexity, University of Colorado, Boulder, Colorado 80309-0216
}

(October 15, 2018)

\begin{abstract}
We study hysteresis for a two-dimensional, spin-1/2, nearest-neighbor, kinetic Ising ferromagnet in an oscillating field, using Monte Carlo simulations and analytical theory. Attention is focused on small systems and weak field amplitudes at a temperature below $T_{c}$. For these restricted parameters, the magnetization switches through random nucleation of a single droplet of spins aligned with the applied field. We analyze the stochastic hysteresis observed in this parameter regime, using timedependent nucleation theory and the theory of variable-rate Markov processes. The theory enables us to accurately predict the results of extensive Monte Carlo simulations, without the use of any adjustable parameters. The stochastic response is qualitatively different from what is observed, either in mean-field models or in simulations of larger spatially extended systems. We consider the frequency dependence of the probability density for the hysteresis-loop area and show that its average slowly crosses over to a logarithmic decay with frequency and amplitude for asymptotically low frequencies. Both the average loop area and the residence-time distributions for the magnetization show evidence of stochastic resonance. We also demonstrate a connection between the residencetime distributions and the power spectral densities of the magnetization time series. In addition to their significance for the interpretation of recent experiments in condensed-matter physics, including studies of switching in ferromagnetic and ferroelectric nanoparticles and ultrathin films, our results are relevant to the general theory of periodically driven arrays of coupled, bistable systems with stochastic noise.
\end{abstract}

PACS number(s): 75.60.-d, 77.80.Dj, 64.60.Qb, 05.40.+j

\section{INTRODUCTION}

Hysteretic response to an oscillating control parameter or "force" is a nonlinear nonequilibrium phenomenon commonly observed in both natural and man-made systems. The example most familiar in physics and electrical engineering is probably the hysteresis loop produced when a ferromagnet at a temperature below its critical temperature $T_{c}$ is placed in an oscillating magnetic field [1 3 . Similar behavior is seen in ferroelectrics 绝 7]. Some other examples are electrochemical adsorbate layers that are driven through a phase transition by an oscillating electrode potential in a Cyclic Voltammetry experiment 88.96, systems driven through a phase transition between different liquid-crystalline phases by pressure oscillations [10], and systems driven through a solid-liquid phase transition by temperature oscillations. Hysteresis is often modeled by systems of differential equations that display discontinuous bifurcations [11] 13].

Systems that exhibit hysteresis have in common a nonlinear, irreversible response, which causes the phase of the response to lag behind the force. The physical mechanism that causes the hysteretic behavior can, however, be quite different in different systems and even in different parameter regimes for the same system. The details of this mechanism must be considered in order to accurately predict such aspects of the hysteretic response as its dependence on the frequency and amplitude of the oscillating force. Here we present a study of hysteresis in a particular model system which incorporates both spatial degrees of freedom and thermal fluctuations - a kinetic Ising ferromagnet in a parameter regime where the model has a first-order phase transition in equilibrium and the system response is stochastic. The model and its behavior in this regime are relevant to at least two different research areas that are rarely discussed together: experimental studies of switching dynamics in nanoscale ferromagnetic and ferroelectric particles and ultrathin films, and theoretical and experimental studies of stochastic resonance in spatially extended systems. We hope the present study may contribute to some intellectual cross-fertilization.

In recent years new experimental techniques, such as magnetic force microscopy (MFM) 114 18, have been developed that permit measurements of the magnetization state and switching behavior of particles as small as a few nanometers. Ferromagnetic particles in this size range consist of a single domain in equilibrium, and together with ultrathin films they are of interest as potential materials for ultra-high density recording media. The dynamics of magnetization reversal in such systems has been modeled with kinetic Ising systems subject to sudden field reversal 
1923. These numerical and analytical studies have given results in qualitative agreement with the experiments mentioned above. Recent experiments on ultrathin ferromagnetic Fe/Au (001) films [24 have considered the frequency dependence of hysteresis loop areas, which were interpreted in terms of effective exponents consistent with those found for a continuous spin model [7]25 27]. Similar experiments on ultrathin $\mathrm{Co}$ films on $\mathrm{Cu}(001)$ have found exponents consistent with a mean-field treatment of the Ising model [28]. These studies of nanoparticles and ultrathin films suggest that experiments can now be performed on systems sufficiently small that atomic-scale simulations become feasible, and that kinetic Ising systems are useful models for switching in such nanoscopic systems.

Since its introduction as a possible model for the time dependence of the Earth's ice ages [29], the concept of stochastic resonance has been applied to a variety of phenomena in physical and biological science and engineering, in which response to a periodic force is enhanced by noise 30]. Most early treatments considered a single bistable element similar to a mean-field model of a ferromagnet 31] 33], with added noise. However, more recently experimental studies have been conducted with chains of coupled diode resonators [34], and numerical and theoretical studies have considered locally coupled one-dimensional time-dependent Ginzburg-Landau or Frenkel-Kontorova models [35 38], Ising models in one [39], two [40], and three [41] dimensions, chains of coupled nonlinear maps [42], and systems of globally coupled bistable elements [43].

Here we consider hysteresis in a two-dimensional spin-1/2, nearest-neighbor, kinetic Ising ferromagnet in an oscillating field with periodic boundary conditions. For convenience, and because of the many experimental measurements of hysteresis that address magnetic systems, we use the customary magnetic language, in which the order parameter is the magnetization per site, $m(t) \in[-1,+1]$, and the force is the magnetic field $H(t)$. However, we expect our results also to apply to stochastic hysteresis phenomena in other areas of science. For example, in dielectrics $m(t)$ and $H(t)$ can be re-interpreted as polarization and electric field, in adsorption problems as coverage $\theta(t)=[2 m(t)-1]$ and (electro)chemical potential or (osmotic)pressure, etc.

Below $T_{c}$ and in zero field this Ising model has two degenerate magnetized phases corresponding to a majority of the spins in the positive or the negative direction. A weak applied field breaks the degeneracy, and the phase with the spins aligned (anti-aligned) with the field is stable (metastable). If the field varies periodically in time, the system is driven back and forth across a first-order phase transition, and the two phases alternate between being momentarily stable and metastable. As a result, $m(t)$ lags behind $H(t)$, and hysteresis occurs. In the regime of small system size, weak applied field, and temperature well below $T_{c}$ considered here, the system switches abruptly and stochastically between the two magnetized phases. A difference between two-dimensional, locally coupled bistable systems, such as this Ising model, and the one-dimensional arrays studied in most of the stochastic-resonance studies cited above [34 39,42], is that locally coupled one-dimensional systems have no ordered phase at nonzero temperature or noise intensity. The apparent long-range order in those studies is therefore a finite-size effect. However, the average equilibrium domain size grows exponentially with decreasing temperature [39,44]. For chains much shorter than this size, the absence of true long-range order should not be qualitatively significant for the hysteretic behavior.

The metastable phase in Ising models exposed to a static field $H$ decays by different mechanisms, depending on the magnitude of $H$, the system size $L$, and the temperature $T$ 445. Two distinct regimes are separated by a crossover field called the dynamic spinodal, $H_{\mathrm{DSP}}(T, L)$. These two decay regimes can be distinguished by the statistical properties of the lifetime of the metastable phase. The lifetime is defined as $\tau=t(m=0)$, the first-passage time to a magnetization of zero, following an instantaneous field reversal from $H$ to $-H$. For $|H| \gg H_{\mathrm{DSP}}$, the mean of the lifetime, $\langle\tau\rangle$, is much greater than its standard deviation, $\sigma_{\tau}$. Therefore, this field region is termed the "deterministic regime." For $|H| \ll H_{\mathrm{DSP}},\langle\tau\rangle \approx \sigma_{\tau}$ and this field region is therefore termed the "stochastic regime." Both the deterministic and stochastic regimes are further subdivided according to the modes by which the metastable phase decays. The deterministic regime is split into the multi-droplet (MD) and strong-field (SF) regions for the low and high fields in this regime respectively. For a given system size the stochastic regime is also divided into the coexistence $(\mathrm{CE})$ and single-droplet (SD) region for the low and high fields in this regime, respectively. Detailed discussions of these different decay modes are found in Refs. 45 47. At sufficiently low $T$ that the single-phase correlation lengths are microscopic, the different decay regimes can be distinguished by the interplay among four length scales: the lattice spacing $a$, the system size $L$, the radius of a critical droplet $R_{c}$, and the average distance between supercritical droplets $R_{0}$. The latter two lengths increase with decreasing field strength: $R_{c} \propto 1 /|H|$ and $R_{0} \propto \exp \left[\right.$ const. $\left./|H|^{d-1}\right]$, where $d$ is the spatial dimensionality. Here we consider specifically decay in the SD region, which is characterized by

$$
a \ll R_{c} \ll L \ll R_{0} .
$$

In this regime, the decay of the metastable phase proceeds by random homogeneous nucleation of a single critical droplet of the stable phase, which then quickly grows to take over the system. We have previously proposed [19, 21, 46] that this decay mechanism may apply to, e.g., barium ferrite particles in the 50-70 nm diameter range [15]. The crossover to the MD region corresponds to $R_{0} \sim L$. As a result, the dynamic spinodal depends asymptotically on $L$ as $H_{\mathrm{DSP}}(T, L) \sim(\ln L)^{-1 /(d-1)}$. In the $\mathrm{SD}$ region the critical droplet is much smaller than the system itself, and 
the crossover to the $\mathrm{CE}$ region is marked by $R_{c} \sim L$. The corresponding crossover field, called the thermodynamic spinodal, therefore depends on $L$ as $H_{\mathrm{THSP}}(T, L) \sim L^{-1}$. In recent exploratory studies 48 [0] we have shown that the response of a kinetic Ising model to an oscillating field is qualitatively different for the MD and SD regions. The details of the response in the MD region will be described in forthcoming papers [51].

Theoretical studies of hysteresis have been performed for several models, using a variety of methods. These include various studies of models with a single degree of freedom, equivalent to mean-field treatments of the Ising model [31, 33., Monte Carlo (MC) simulations of the spin-1/2 Ising model 26,27,52 60], and several $O(N)$ type models [7,25 227,61. These studies were performed with variations in the details of the simulations and in the model parameters. Most of them indicate that the average hysteresis-loop area, $\langle A\rangle=-\langle\oint m(H) d H\rangle$, appears to display power-law dependences on the frequency and amplitude of $H(t)$. However, there is no universal agreement on the values of the exponents, either experimentally or theoretically. For the Ising model, nucleation effects that would lead to a logarithmic frequency dependence have been proposed [6,26]62]. A mean-field model exhibits a dynamic phase transition in which the mean period-averaged magnetization, $\langle Q\rangle=(\omega / 2 \pi)\langle\oint m(t) d t\rangle$, changes from $\langle Q\rangle \neq 0$ to $\langle Q\rangle=0$ [32. Such a dynamic phase transition has been suggested from MC simulations of a kinetic Ising model as well [52 [57,59,60, 63].

The work presented in this paper differs from most past theoretical and numerical studies of hysteresis in two important ways. First, mean-field models do not take into account thermal noise and spatial variations in the order parameter, thus ignoring fluctuations which may be important in real materials. Second, most previous investigations of hysteresis in Ising models have considered the frequency and amplitude dependence of quantities such as $Q$ and $A$, without considering the manner in which the metastable phase decays.

Considering the nucleation-based single-droplet decay mechanism, we find that the average hysteresis-loop area exhibits an extremely slow crossover to a logarithmic decay with frequency and amplitude in the asymptotic lowfrequency limit. This crossover is sufficiently slow that the behavior can easily be misinterpreted as a power law over several orders of magnitude in frequency. We also show that the average loop area and the residence-time distributions for the system magnetization exhibit evidence of stochastic resonance, and we provide a connection between the characteristic decay time of the residence-time distributions and the power spectral densities of the magnetization time series. We find no evidence of a dynamic phase transition in the SD region.

The rest of this paper is organized as follows. Section II supplies background information on the simulation of the kinetic Ising model. In Sec. III some general properties of the time-series data are discussed. In Sec. IV the probability that the system magnetization does not switch sign during a period of the field, $P_{\text {not }}(\omega)$, is derived. This derivation is central to theoretical calculations throughout this paper. Section $\nabla$ presents theoretical calculations and $\mathrm{MC}$ simulation data for the residence-time distributions (RTDs). Also, we define and calculate the characteristic time of the RTDs and show its relevance to the low-frequency behavior of the power spectral densities (PSDs) of the time series, which are analyzed in Sec. VI. Section VII discusses the hysteresis-loop area, the correlation between the magnetization and the field, and the period-averaged magnetization. Finally, Sec. VIII contains a summary and conclusions.

\section{MODEL}

The model used in this study is a kinetic, nearest-neighbor Ising ferromagnet on a hypercubic lattice with periodic boundary conditions. The Hamiltonian is given by

$$
\mathcal{H}=-J \sum_{\langle i j\rangle} s_{i} s_{j}-H(t) \sum_{i} s_{i}
$$

where $s_{i}$ is the state of the $i$ th spin and can have the values $s_{i}= \pm 1, \sum_{\langle i j\rangle}$ runs over all nearest-neighbor pairs, and $\sum_{i}$ runs over all $N=L^{d}$ lattice sites. The order parameter is the time-dependent magnetization per site,

$$
m(t)=\frac{1}{L^{d}} \sum_{i=1}^{N} s_{i}(t) .
$$

The dynamic used is the Glauber [44] single-spin-flip Monte Carlo algorithm with updates at randomly chosen sites. The time unit is one Monte Carlo step per spin (MCSS). The system is put in contact with a heat bath at temperature $T$, and each attempted spin flip from $s_{i}$ to $-s_{i}$ is accepted with probability 64.

$$
W\left(s_{i} \rightarrow-s_{i}\right)=\frac{\exp \left(-\beta \Delta E_{i}\right)}{1+\exp \left(-\beta \Delta E_{i}\right)} .
$$


Here $\Delta E_{i}$ is the change in the energy of the system that would result if the spin flip were accepted, and $\beta=1 / k_{\mathrm{B}} T$ where $k_{\mathrm{B}}$ is Boltzmann's constant. It has been shown in the weak-coupling limit that the stochastic Glauber dynamic can be derived from a quantum-mechanical Hamiltonian in contact with a thermal heat bath modeled as a collection of quasi-free Fermi fields in thermal equilibrium [65].

In this paper, all numerical calculations are performed for $d=2, L=64$ and $T=0.8 T_{c}$. This value of $T$ is sufficiently far away from the critical temperature so that the thermal correlation length is small compared to the critical droplet radius and the size of the system. The system is subject to either an oscillating field, $H(t)=-H_{0} \sin (\omega t)$, or to a constant field of magnitude $H_{0}$.

As discussed in Sec. I, the decay of the metastable phase in the presence of an external field $H$ proceeds by nucleation of droplets of the stable phase [45]. Figure 1 s shows the metastable and stable phases as local minima in the free energy,

$$
F(m, H, T)=F(m, 0, T)-m L^{2} H,
$$

of a nearest-neighbor Ising model on a $64 \times 64$ lattice at $T=0.8 T_{c}$ 66, 67. For $H=0$ there are two degenerate equilibrium phases of magnetization $\pm m_{\mathrm{eq}}(T)$, separated by a free-energy barrier of height proportional to $L^{d-1}$. For $H=H_{0}=0.1 J$ the value of $m$ near +1 , where $F$ has its global minimum, is the stable magnetization. The local minimum near $m=-1$ represents the metastable phase. The convex parts of the barrier represent a single spherical droplet of one phase embedded in the other. The droplet is a collective excitation [43] through which the switching proceeds, and the critical droplet is the droplet configuration corresponding to the local maximum of $F$ at a given value of $H$ 66. For $H<0$ the stable and metastable phases are reversed.

The average number of droplets of the stable phase that are formed per unit time and volume is given by the field and temperature dependent nucleation rate,

$$
I(H(t), T) \approx B(T)|H(t)|^{K} \exp \left[-\frac{\Xi_{0}(T)}{|H(t)|^{d-1}}\right] .
$$

The notation follows that of Ref. [19], where $B(T)$ is a non-universal temperature dependent prefactor, and $K$ and $\Xi_{0}(T)$ are known from field theory [68 [70] and simulations [45] and are listed in Table [. The quantity $\Xi_{0}(T)$ is the field-independent part of the free-energy cost of a critical droplet, divided by $k_{B} T$. The external field, $H(t)$, is the only quantity through which $I(H(t), T)$ depends on time in this adiabatic approximation.

Several quantities, whose values do not depend on the frequency of the applied field, are required as input for the theoretical calculations in the following sections. These quantities, which include the average lifetime $\left\langle\tau\left(H_{0}\right)\right\rangle \approx$ $\left[L^{d} I\left(H_{0}, T\right)\right]^{-1}$, are listed in Table $\mathbb{0}$. They are determined through what we refer to as "field-reversal simulations." In these simulations the system initially has all spins up or positive. It is then subjected to a static external field of magnitude $H_{0}$ with a sign opposite the system magnetization. This instantaneous field quench prepares the system in a metastable state, and the decay of the metastable phase proceeds by the mechanisms outlined in the introduction.

\section{TIME-SERIES DATA}

In the simulations presented here, a sinusoidal field is applied to the system. Its amplitude, $H_{0}=0.1 J<H_{\mathrm{DSP}}$, is chosen such that in field-reversal simulations the system is clearly in the SD region for a field of magnitude $H_{0}$. The dynamic spinodal field is approximated by $H_{\mathrm{DSP}} \approx H_{1 / 2}$, where $H_{1 / 2}$ is the value of $H$ (for given $L$ and $T$ ) for which the relative standard deviation of the lifetime, $r=\sigma_{\tau} /\langle\tau\rangle$, is $1 / 2$ [45]. This value of $H_{\mathrm{DSP}}$ is given in Table 1 . It approximately equals the field for which the local minimum in Fig. 1 disappears [66].

To obtain the raw time-series data, an Ising system was initially prepared with either a random arrangement of up and down spins with $m(t=0) \approx 0$, or with a uniform arrangement with all spins up. Then the sinusoidal field, $H(t)=-H_{0} \sin (\omega t)$, was applied and changed every attempted spin flip, allowing for a smooth variation of the field. The time series did not appear to depend on the initial conditions after a few periods. The simulations were performed with several values of the driving frequency $\omega$. For each frequency, we recorded the time-dependent magnetization $m(t)$ for approximately $16.9 \times 10^{6}$ MCSS. Each of these raw time-series data files store the values of $t, H(t)$, and $m(t)$ in increments of 1 MCSS. Each file takes up about 800 megabytes and took about 9 days to run, using a single node of an IBM sp2. These are by far the most extensive MC simulations of hysteresis in Ising systems to date.

It is useful to think of hysteresis as a competition between two time scales: the average lifetime of the metastable state following an instantaneous field reversal from $H_{0}$ to $-H_{0},\left\langle\tau\left(H_{0}\right)\right\rangle$, and the period of the external forcing field, $2 \pi / \omega$. Therefore, we specify the ratio $R$ of the period to the average lifetime,

$$
R=\frac{(2 \pi / \omega)}{\left\langle\tau\left(H_{0}\right)\right\rangle}
$$


One may think of $R(1 / R)$ as a scaled period (scaled frequency).

We note that $\left\langle\tau\left(H_{0}\right)\right\rangle$ is the "shortest of the long time scales" in the present system. From Fig. 1 we observe that whereas the free-energy barrier represented by the critical droplet for $|H|=H_{0}$ is on the order of $k_{B} T$, the barrier between the degenerate phases at $H=0$ is on the order of $50 k_{B} T$. For this temperature and system size, the timescale for spontaneous fluctuations between the phases in the absence of an applied field, $\langle\tau(0)\rangle$, is therefore essentially infinite. Conversely, the nucleation of the critical droplet necessary to leave the metastable phase is entirely driven by the thermal fluctuations, even when the field has its maximum strength $H_{0}$. Switching in this system therefore truly depends on the joint action of the random thermal noise and the deterministic oscillating field.

Figure 2 shows short initial segments of the magnetization time series in the SD region for three different values of $R$. In all three cases, $m(t)$ fluctuates near one of the two degenerate values of the spontaneous zero-field magnetization, punctuated by rapid transitions between these two values, that are completed during a single half-period of the applied field. The rapid switching of $m(t)$ is evidence of the nucleation of a single critical droplet that reverses the sign of the magnetization. The magnetization 'plateaus' are due to the failure of any critical droplet of the stable phase to nucleate. The fluctuations in $m(t)$ on these plateaus indicate appearance and disappearance of sub-critical droplets. For low driving frequencies the magnetization switches twice during almost every field cycle, whereas for high frequencies switching occurs only occasionally. The 'spikes' in $m(t)$ seen for $R=2.5$ occur when a single droplet nucleates, but does not have time to grow and switch the system magnetization before the field becomes unfavorable and the droplet rapidly collapses. The number of field cycles shown in Fig. 2 is small compared to the total number of cycles in an entire time series.

\section{PROBABILITY OF NOT SWITCHING DURING A PERIOD}

The probability that the system does not switch during a full period of the field, $P_{\text {not }}(\omega)$, is central to the theoretical understanding of hysteresis in the SD region. It occurs most directly in the calculation of the residence-time distributions in Sec. V. In addition, elements in the derivation of $P_{\text {not }}(\omega)$ are fundamental for describing most of the observed quantities in this study.

As mentioned in Sec. III, the system exhibits abrupt switches during which the average magnetization changes between values near $\pm m_{\mathrm{eq}}$. As seen in Fig. 2, these events occur quickly compared to the period of the external field, and to a first approximation the time it takes the droplet to grow to fill the system (the growth time) is negligible. A more realistic treatment takes the finite growth time into account as a lag time between the nucleation of a critical droplet and the time at which the system switches.

The first part of the derivation is presented without the effects of the growth time. This is done for simplicity, as well as to emphasize the role of the growth time as a correction to the basic picture of a variable-rate Poisson process. First we derive the expression for the cumulative probability that a switching event has occurred by time $t, F(t)$, in terms of the time-dependent rate of an instantaneous decay process, $\rho(t)$. [This cumulative probability should not be confused with the free energy $F(m, H, T)$ of Eq. (2.4).] It is convenient to introduce $\bar{F}(t)=1-F(t)$, the probability that a switching event has not occurred by time $t$. Standard theory of variable-rate Markov processes [73] leads to a difference equation for $\bar{F}(t)$,

$$
\begin{aligned}
\bar{F}(t+\Delta t) & =\bar{F}(t) \times\{\text { probability an event has not occurred in the interval }[t, t+\Delta t]\} \\
& =\bar{F}(t)[1-\rho(t) \Delta t]
\end{aligned}
$$

which in the limit $\Delta t \rightarrow 0$ gives

$$
d \bar{F}(t) / d t=-\rho(t) \bar{F}(t) .
$$

The growth time, $t_{\mathrm{g}}(t)$, is introduced into the derivation at the level of Eq. (4.1). It is defined as the time between the nucleation of a critical droplet and the time when the volume of this droplet becomes approximately half the system volume. The dependence of the growth time on $t$ is a consequence of the time dependence of the interface growth velocity, which is approximately proportional to $H(t)$. For suitably long time scales, the growth of a supercritical droplet is a deterministic process. Another quantity in this derivation is the time at which a droplet nucleates, $t_{\mathrm{n}}(t)$. If a switching event occurs at time $t$, then $t_{\mathrm{n}}(t)=t-t_{\mathrm{g}}(t)$. Where clarity is not sacrificed, we do not show the explicit $t$ dependence of $t_{\mathrm{g}}$ or $t_{\mathrm{n}}$. For a switching event to occur in any particular period of the external field, a critical droplet must not only nucleate, but must do so early enough so there is sufficient time for it to grow to the volume of the system. Therefore, the difference equation for $\bar{F}(t)$ is modified to read 


$$
\begin{aligned}
\bar{F}(t+\Delta t) & =\bar{F}(t) \times\{\text { probability a switch has not occurred within }[t, t+\Delta t]\} \\
& =\bar{F}(t) \times\left\{\text { probability a droplet has not nucleated within }\left[t_{\mathrm{n}}, t_{\mathrm{n}}+\Delta t_{\mathrm{n}}\right]\right\} \\
& =\bar{F}(t)\left[1-\rho\left(t_{\mathrm{n}}\right) \Delta t_{\mathrm{n}}\right] \\
& =\bar{F}(t)\left[1-\rho\left(t_{\mathrm{n}}\right) \frac{d t_{\mathrm{n}}}{d t} \Delta t\right] .
\end{aligned}
$$

We can express this result in terms of the growth time $t_{\mathrm{g}}$ and its derivative, using

$$
\frac{d t_{\mathrm{n}}}{d t}=1-\frac{d t_{\mathrm{g}}}{d t}
$$

Substituting into Eq. (4.3) and letting $\Delta t \rightarrow 0$ gives

$$
\frac{d \bar{F}(t)}{d t}=-\rho\left(t-t_{\mathrm{g}}\right)\left[1-\frac{d t_{\mathrm{g}}}{d t}\right] \bar{F}(t) .
$$

Integrating Eq. (4.5) gives the cumulative distribution,

$$
F(t)=1-\exp \left[-\int_{0}^{t} \rho\left[t^{\prime}-t_{\mathrm{g}}\left(t^{\prime}\right)\right]\left(1-\frac{d t_{\mathrm{g}}\left(t^{\prime}\right)}{d t^{\prime}}\right) d t^{\prime}\right] .
$$

Differentiation gives the probability density function (pdf) for switching events at time $t$,

$$
P(t)=\frac{d F}{d t}=\rho\left[t-t_{\mathrm{g}}(t)\right]\left[1-\frac{d t_{\mathrm{g}}(t)}{d t}\right] \exp \left[-\int_{0}^{t} \rho\left[t^{\prime}-t_{\mathrm{g}}\left(t^{\prime}\right)\right]\left(1-\frac{d t_{\mathrm{g}}\left(t^{\prime}\right)}{d t^{\prime}}\right) d t^{\prime}\right] .
$$

For $t_{\mathrm{g}}=0$, Eq. (4.7) is equivalent to Eq. (9) of 74]. The growth time $t_{\mathrm{g}}$ is obtained from the expression for the time-dependent volume of a supercritical droplet

$$
V\left(t, t_{\mathrm{n}}\right)=\Omega\left[\int_{t_{\mathrm{n}}}^{t} v\left(t^{\prime}\right) d t^{\prime}\right]^{d},
$$

where $t>t_{\mathrm{n}}$. Here $v(t)$ is the droplet interface velocity and $\Omega$ is defined such that the volume of an equilibrium droplet of radius $R$ is $\Omega R^{d}$ 771]. Using the Lifshitz-Allen-Cahn approximation [75 78], the interface velocity is $v(t) \approx \nu|H(t)|$. The proportionality constant $\nu$ depends on the details of the dynamics. Here we use values for the Glauber dynamics, obtained from field-reversal simulations by Ramos et al. [72]. The values of the constants used in our calculations are listed in Table 1 . For $d=2$, Eq. (4.8) for the growth time becomes,

$$
\begin{aligned}
V\left(t, t-t_{\mathrm{g}}\right)=\frac{L^{2}}{2} & =\Omega\left[\int_{t-t_{\mathrm{g}}}^{t} \nu H_{0} \sin \omega t^{\prime} d t^{\prime}\right]^{2} \\
& =\frac{\Omega \nu^{2} H_{0}^{2}}{\omega^{2}}\left\{\cos \omega\left[t-t_{\mathrm{g}}\right]-\cos \omega t\right\}^{2}
\end{aligned}
$$

For a static field of strength $H_{0}$, the growth time is

$$
\tilde{t}_{\mathrm{g}}=\frac{1}{\sqrt{2 \Omega}}\left(\frac{L}{\nu H_{0}}\right) .
$$

Substituting this expression into Eq. (4.9) gives

$$
\cos \omega\left[t-t_{\mathrm{g}}\right]=\tilde{t}_{\mathrm{g}} \omega+\cos \omega t .
$$

Solving for $t_{\mathrm{g}}$ such that $t_{\mathrm{g}}<t$ gives

$$
t_{\mathrm{g}}(t)= \begin{cases}t-\left\{\frac{1}{\omega} \cos ^{-1}\left[\cos \omega t+\tilde{t}_{\mathrm{g}} \omega\right]\right\} & t_{0}<t<\pi / \omega \\ 0 & \text { otherwise }\end{cases}
$$

where 


$$
t_{0}=\frac{1}{\omega} \cos ^{-1}\left[1-\tilde{t}_{\mathrm{g}} \omega\right] .
$$

The time $t_{0}$ is the first time during a period for which the probability of switching is non-zero. If $H(t=0)=0$ and $m(t=0) \approx-1$, then the probability density $P(t)$ that a switching event takes place at time $t$ is

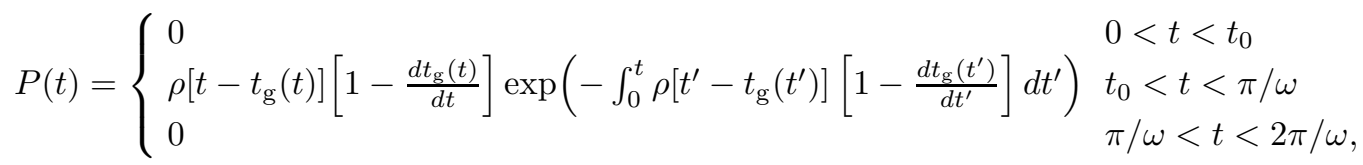

where the ranges for $t$ ensure $P(t)$ is non-zero only when $t>t_{\mathrm{g}}$ and the signs of $m(t)$ and $H(t)$ are not equal. Higherorder corrections, including the probability that a second droplet nucleates during $t_{\mathrm{g}}$, were found to be numerically insignificant. The main approximation used here to obtain $t_{g}(t)$ lies in ignoring the slower growth of droplets only slightly larger than the critical radius. This has been shown to be permissible for adiabatically slow-forcing models in which large droplets grow exponentially in time [79]. In the present case, however, we simply consider it a convenient approximation, whose accuracy is ultimately confirmed by our numerical simulations.

In the SD region, the average lifetime in a field-reversal simulation should be dominated by nucleation. Therefore, the total nucleation rate in a static field $H_{0}$ can be expressed as

$$
\begin{aligned}
\rho_{0} & =\left[\langle\tau\rangle-\tilde{t}_{\mathrm{g}}\right]^{-1} \\
& =L^{d} I\left(H_{0}, T\right) .
\end{aligned}
$$

The nucleation rate in a static field of strength $H_{0}$ should be equal to the nucleation rate in a sinusoidal field of amplitude $H_{0}$ at the maximum of the field, $\rho_{0}=\rho(t=\pi / 2 \omega)$. The ratio of these two decay rates is then

$$
\frac{\rho(t)}{\rho_{0}}=\frac{I(H(t), T)}{I\left(H_{0}, T\right)} .
$$

Substituting the form of the nucleation rate, Eq. (2.5), into the expression above allows $\rho(t)$ to be recast in a form which does not explicitly contain the non-universal prefactor $B(T)$ :

$$
\rho(t)=\rho_{0}|\sin (\omega t)|^{K} \exp \left[-\frac{\Xi_{0}(T)}{\left|H_{0}\right|^{d-1}}\left(\frac{1}{|\sin (\omega t)|^{d-1}}-1\right)\right] .
$$

This expression holds when $m(t)$ and $H(t)$ have opposite signs, while $\rho(t)=0$ when they have the same sign. Using Eq. (4.15a) for the maximum decay rate gives $\rho_{0}=(6.62 \pm 0.07) \times 10^{-4}$ MCSS $^{-1}$, using quantities listed in Table [1. Figure 3 shows $P(t) / \omega$ vs. $\omega t$ for five different frequencies of the external field. The inset in Fig. 3 shows $\rho(t)$ vs. $\omega t$. The nucleation rate achieves its maximum value, $\rho_{0}$, at a phase of $\omega t=\pi / 2$, independent of $\omega$. However, the location and width of the maximum for $P(t)$ depend strongly on $\omega$. This behavior results from the combined field dependence of the nucleation rate and the interface growth velocity. For $R \gtrsim 100, P(t)$ narrows and the location of its maximum shifts to lower phase values as the switching begins to occur before the maximum in $\rho(t)$. As $R$ is decreased below 1.5, $\omega t_{0} \rightarrow \pi$, and the area under the curve for $P(t) / \omega$ goes to zero. Therefore, the condition $\omega_{\max } t_{0}=\pi$ gives the maximum frequency for which single-droplet switching is possible. Using Eq. (4.13) and converting the result to a bound on $1 / R$ gives $(1 / R)_{\max }=1.19 \pm 0.03$. For higher frequencies, switching events are very rare, and if they occur at all, they do so through a multidroplet mechanism. The probability of not switching during an entire period is obtained by integrating the probability density $P(t)$,

$$
\begin{aligned}
P_{\text {not }}(\omega) & =1-\int_{0}^{\frac{\pi}{\omega}} P\left(t^{\prime}\right) d t^{\prime} \\
& =\bar{F}\left(\frac{\pi}{\omega}\right) .
\end{aligned}
$$

The frequency dependence of $P_{\text {not }}(\omega)$ is the aspect of this quantity most important for comparisons with our MC data. However, $P_{\text {not }}(\omega)$ also depends on other parameters through the nucleation rate.

\section{RESIDENCE-TIME ANALYSIS}

As mentioned in Sec. III, the magnetization exhibits abrupt switches between values near $\pm m_{\mathrm{eq}}$. In contrast, the times between the magnetization reversals are comparable to, or greater than, the period of the applied field. As a 
first approximation, one may therefore consider these switching events as occurring in a discrete two-state system. For an Ising system undergoing a field-reversal experiment [45], the lifetime in the SD region is stochastic and is well described by droplet theory. For an oscillating field, the analogous quantity is the time between reversals of the magnetization, called the residence time. The probability density for the residence times is called the residence-time distribution (RTD) [80,81]. In Sec. VA we construct analytical expressions for the RTDs and compare these with the RTDs obtained from our simulated time series. In Sec. VB we calculate the area of the peaks in the RTDs, or the RTD peak strengths, and compare our theoretical results for the peak strengths with MC data. Finally, we show that our data for the RTD peak strengths provide evidence of stochastic resonance in the model.

\section{A. Residence-time distributions}

We define the residence time, $\Delta$, as the time between consecutive magnetization reversals, and denote its probability density as $\Pi(\Delta)$. The details of our theoretical deviation of $\Pi(\Delta)$ are given in Appendix $\mathrm{A}$. The results of the theoretical calculation for the residence-time distributions, which contain no adjustable parameters, are shown as solid curves in Fig. 1(a-c) for different values of $R$.

Next, we give a description of the MC analysis for the RTDs. (The results of this analysis are shown as solid dots in Fig. $1(\mathrm{a}-\mathrm{c})$ for different values of $R$.) When measuring an RTD one must ignore "false crossing events." In these events, the magnetization crosses zero and re-crosses zero again within a short time without having reached a value near the stable magnetization. There appear to be two reasons for these re-crossing events. First, when $m(t) \approx 0$ the magnetization can re-cross zero many times due to thermal fluctuations. Second, during some of the periods there will be a "spike" in the magnetization when a supercritical droplet nucleates but does not have time to completely take over the system before the applied field changes sign. For this reason, a cutoff is employed, and a switching event is recorded only when $m(t)$ reaches some cutoff value $\pm m_{\text {cut }}$. To quantify the meaning of the cutoff, define $t_{i}^{+}$and $t_{i}^{-}$ as the times at which $m\left(t_{i}^{+}\right)=+m_{\text {cut }}$ and $m\left(t_{i}^{-}\right)=-m_{\text {cut }}$, respectively. The residence time $\Delta_{i}$ is given by

$$
\Delta_{i}=\left\{\begin{array}{lll}
t_{i+1}^{+}-t_{i}^{-} & \text {when } m(t) \approx-1 \text { for } t_{i}^{-}<t<t_{i+1}^{+} \\
t_{i+1}^{-}-t_{i}^{+} & \text {when } m(t) \approx+1 \text { for } t_{i}^{+}<t<t_{i+1}^{-}
\end{array} .\right.
$$

We used $m_{\text {cut }}=0.25$. For each frequency, the residence times are measured over an entire time series. The size of the bins in an RTD is set by dividing the maximum observed residence time by the number of bins. Both the maximum residence time for a given time series and the number of bins are different for different frequencies. Hence, the size

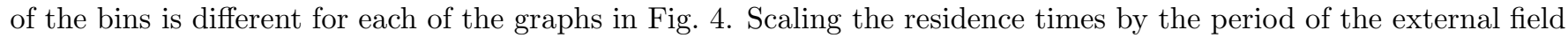
centers the peaks in the RTD about every odd half-integer. In the low-frequency limit, the system spends enough time in an unfavorable field during every half-period to allow the magnetization to switch. In this limit, the RTD would contain a single peak centered around $1 / 2$. As the frequency of the field is increased, there should be more periods during which the magnetization does not switch at all, indicated in the RTDs by an increase of the size of the peaks centered on $3 / 2,5 / 2$, etc. The RTD data from MC simulations are shown in Fig. 1 as solid points together with the theoretical curves.

The smoothest MC results, and the best agreement between theory and simulation occur for $R \gtrsim 10$. The agreement is quite good, considering that the theoretical calculation contains no free parameters. All of the constants in the formulas for the nucleation rate and the interface growth velocity come from theoretical considerations or from fieldreversal simulations. The agreement is poor only for the highest frequencies, corresponding to $R \lesssim 2.5$. For these values of $R$ the MC data are suspect. First, for these high frequencies of the external field, the sizes of the peaks for large residence times are significant. For all of the frequencies shown, the data sets have approximately the same total number of switching events. Therefore, a smaller number of events is contained in each bin of the RTDs for the higher frequencies. Second, in spite of the cutoff there are two peaks in the RTDs for $R=2$ through 5 in the interval $0<\omega \Delta / 2 \pi<1$. Of these two peaks, the peak at the shorter residence time comes from "spikes" in the magnetization which are large enough to extend past the cutoff value, but still do not switch the system completely within a field period. These "spikes" in the time series redistribute weight from the higher-order peaks of the RTD into the spurious peak at short residence times. This affects the measurement of the peak strengths from the MC data as well, as discussed in the next section.

\section{B. RTD peak strengths}

The frequency dependence of the strengths of the peaks in the RTD is another quantity which describes the nature of the magnetization reversal. The strength of the $j$-th peak in an RTD is given by 


$$
S_{j}(\omega)=\int_{(j-1) \frac{2 \pi}{\omega}}^{j \frac{2 \pi}{\omega}} \Pi(\Delta) d \Delta .
$$

The peak strengths obtained from the MC time series are shown as solid dots in Fig. 5. The statistical errors are estimated by error-propagation analysis as $\sqrt{S_{j}\left(1-S_{j}\right) / N}$, where $N$ is the total number of switching events in a simulation run. For almost all of the data points the error bars are smaller than the symbol size. The solid lines in Fig. 5 are theoretical results. The strength of the first peak, $S_{1}(\omega)$, is simply the probability that $m(t)$ switches sign within the first period after the last field reversal,

$$
S_{1}(\omega)=1-P_{\text {not }}(\omega) .
$$

Therefore, the strength of the $j$-th peak is

$$
S_{j}(\omega)=P_{\mathrm{not}}(\omega)^{j-1}\left[1-P_{\mathrm{not}}(\omega)\right] .
$$

The values for $P_{\text {not }}(\omega)$ used in this calculation were obtained by numerically integrating Eq. (4.18) for several values of $\omega$. This parameter-free numerical evaluation of the theoretical peak strengths is in good agreement with the MC data. In Fig. f one can see this agreement, especially for the strength in the first peak, $S_{1}(\omega)$, for all but the highestfrequency data point at $1 / R=0.5$. However, the MC data slightly overestimate $S_{1}(\omega)$ even for low frequencies. This is due to the redistribution of strength from the higher-order peaks into the first peak due to "spikes" in the time series which extend past the cutoff, as mentioned in Sec. VA. Hence, the peak strengths for the higher-order peaks are systematically underestimated by the MC data, particularly for $S_{2}(\omega)$. The agreement between the theoretical curve and the data is not quite as good for $S_{2}(\omega), S_{3}(\omega)$, and $S_{4}(\omega)$ at higher frequencies. However this is expected, due to the poorer statistics for these higher-order peaks.

Analysis of the RTDs for two-state systems has been used to detect stochastic resonance (SR) [30,80]. Gammaitoni et al. studied the switching behavior and residence times of an analog circuit, which served as a model of a bistable system driven by random noise and a sinusoidal external forcing [81]. They found for their model that SR is manifest in the fact that each of the peak strengths in the RTDs has a maximum for a given frequency of the field. If the frequency $\omega_{j}$ corresponds to the location of the maximum in $S_{j}$, then

$$
\omega_{1}<\omega_{2}<\omega_{3}<\ldots
$$

This approach gives an alternative definition for SR, different from the original definition as the noise intensity for which the signal-to-noise ratio (SNR) exhibits a maximum [30]. Since the SNR does not exhibit a maximum with respect to the frequency of the forcing, the definition suggested by Gammaitoni et al. facilitates the understanding of SR as the tuning of one timescale (inverse frequency) to another (average lifetime of the metastable phase), more analogous to a "bona fide" resonance.

The case studied in Ref. [81] was one of a very weak oscillating field, such that the noise-driven switching rate in zero field was of the same order of magnitude as the escape rate from the metastable state in maximum field. As a result, there was only one long timescale, and for sufficiently low frequencies most of the escapes were completely thermally driven. This would redistribute the number of escapes with residence times less than $\pi / \omega$ into a peak at much shorter times, corresponding to the thermal escape rate of the unforced bistable system and giving a nonzero value of $\omega_{1}$.

For the system studied here the thermal switching rate in zero field is virtually zero, as pointed out in the discussion following Eq. (3.1). As a result, $S_{1}$ increases monotonically towards unity as the driving frequency is lowered. For $S_{1}$ to display a maximum, the weight in the RTD's must shift towards times much shorter than the period of $H(t)$. These residence times would correspond to events in which a single thermal fluctuation switches the system. One can increase the thermal switching rate in zero field (or decrease the thermal relaxation time in zero field $\langle\tau(0)\rangle$ ) by increasing $T$. (Recent measurements of telegraph noise in nanoscale ferromagnetic particles are able to show this quite clearly [32].) But increasing the temperature can move the system into an entirely different decay regime (either the $\mathrm{MD}$ or SF regime, depending on the value of $T$ ) where the stochastic nature of the response disappears. However, even though $S_{1}$ does not display a maximum, the higher-order peak strengths do have maxima at $\omega_{2}<\omega_{3}<\ldots$ From Eq. (5.4) the theoretical positions of these maxima are seen to be given by the condition $P_{\text {not }}\left(\omega_{j}\right)=(j-1) / j$, which yields $\omega_{1}=0$. Through $P_{\text {not }}(\omega)$ they are determined by the competition between the period of the deterministic forcing and a stochastic timescale, which is the minimum metastable lifetime $\left\langle\tau\left(H_{0}\right)\right\rangle$.

If we were to reduce $L$ to push the simulations into the coexistence $(\mathrm{CE})$ region, then $\langle\tau(0)\rangle$, which depends exponentially on $L^{d-1}$ through the barrier in the free energy $F(m, 0, T)$, would approach the escape time $\left\langle\tau\left(H_{0}\right)\right\rangle$, which by Eq. (4.15b) is inversely proportional to $L^{d}$. As a result, $S_{1}$ should be observed to decrease at very low frequencies for sufficiently small systems. In this regime, the critical droplet volume would be on the order of half 
the system volume. This effect was recently observed by Lindner et al. in simulations of a one-dimensional chain of bistable elements driven at a constant frequency and subject to noise of variable intensity [36],37].

Our results lead us to make the observation that a maximum in $S_{1}$ is not necessary for the response of the system to the oscillating field to be characterized as "resonant." Rather, $m(t)$ is essentially synchronized with $H(t)$ in the whole frequency range where $S_{1}$ is close to unity. The upper limit of this range is proportional to $\omega_{2}$ and therefore determined by $\left\langle\tau\left(H_{0}\right)\right\rangle$, whereas the lower limit would be determined by the (in our case unobservably long) $\langle\tau(0)\rangle$. This theme will be discussed further in Sec. VII A.

\section{Characteristic time of the RTDs}

For each of the RTDs shown in Fig. A, the size of the peaks decreases for large residence times. The rate of this decrease can be quantified by measuring how the peak strengths decrease with increasing peak number. We define the characteristic time, $\eta(\omega)$, for the RTDs (in units of the field period, $2 \pi / \omega$ ) as

$$
\eta(\omega)=\frac{1}{\ln S_{j}(\omega)-\ln S_{j+1}(\omega)} .
$$

The value of $\eta(\omega)$ for any frequency of the field is measured from the MC data by plotting $\ln S_{j}$ vs. $j$. The slope of a best-fit line through this data gives $-1 / \eta(\omega)$. The inverse characteristic times calculated from the MC data by a weighted least-squares fit are shown in Fig. 6 . The statistical uncertainty in the estimates for the characteristic time becomes large for the lowest and highest frequencies. For the very lowest frequency shown, $1 / R=0.05$, few switching events contribute to any of the peaks in the RTD, other than the first peak. Hence there are poor statistics in the $\ln S_{j}$ data for $j>1$, resulting in a large uncertainty in the fitted slope. For high frequencies the RTDs contain many peaks. Since the total number of switching events in each time series is approximately equal, the statistics for each peak is poor. In addition to this purely statistical error, there is also a systematic effect due to the cutoff used to measure the residence times. Namely, the RTDs display two peaks for the interval $0<\omega \Delta / 2 \pi<1$. This extra weight in $S_{1}(\omega)$ introduces additional systematic error which tends to raise the estimates of $|1 / \eta(\omega)|$ for higher frequencies.

The theoretical calculation of the characteristic time starts by substituting Eq. (5.4) for $S_{j}(\omega)$ into Eq. (5.6), which gives

$$
\eta(\omega)=\frac{-1}{\ln P_{\mathrm{not}}(\omega)} .
$$

The calculation of $\eta(\omega)$ is now trivial because $P_{\text {not }}(\omega)$ has already been evaluated numerically in Sec. VB. The solid curve in Fig. 6 shows the theoretical results from the full numerical calculation of $P_{\text {not }}(\omega)$. The theoretical result diverges at a value of $1 / R \approx 1.19$, the maximum value of the frequency for which single-droplet switching is possible. At this value of $1 / R, P_{\text {not }}=1$, and the characteristic time diverges. For frequency values $1 / R>1.19$ mechanisms other than single-droplet decay might be possible, such as several droplets nucleating simultaneously. Given the length of our simulations, it is unlikely such behavior would be observed. Indeed, the statistical and systematic errors discussed above preclude accurate measurement of the characteristic time from the MC data for $1 / R \gtrsim 0.4$.

For low frequencies, both the theoretical and the MC results for the characteristic time appear constant. This motivates the search for an approximate, analytic expression. We start by casting Eq. (5.7) in terms of the cumulative probability distribution,

$$
\eta(\omega)=-\frac{1}{\ln \bar{F}\left(\frac{\pi}{\omega}\right)} .
$$

Using the low-frequency approximation $t_{\mathrm{g}}=0$ gives

$$
\eta(\omega)=\frac{1}{2 \int_{0}^{\frac{\pi}{2 \omega}} \rho\left(t^{\prime}\right) d t^{\prime}},
$$

where the upper limit in the integral is rewritten because the nucleation rate $\rho(t)$ is symmetric about $t=\pi / 2 \omega$. Substituting $u=\sin (\omega t)$ into Eq. (4.17) for $\rho(t)$ allows one to write the integral above as

$$
\eta(\omega)=\left[2 \rho_{0} \frac{1}{\omega} \int_{0}^{1} \frac{u^{3}}{\sqrt{1-u^{2}}} \exp \left[-\frac{\Xi_{0}(T)}{H_{0}}\left(\frac{1}{u}-1\right)\right] d u\right]^{-1} .
$$


The final result for the characteristic time is given in units of $\langle\tau\rangle$ by multiplying both sides of the equation above by $R$ and simplifying,

$$
R \eta=\left[\frac{\rho_{0}\langle\tau\rangle}{\pi} \int_{0}^{1} \frac{u^{3}}{\sqrt{1-u^{2}}} \exp \left[-\frac{\Xi_{0}(T)}{H_{0}}\left(\frac{1}{u}-1\right)\right] d u\right]^{-1},
$$

where all the quantities are known except for the integral, which can be calculated numerically. This theoretical result is shown in Fig. 6 as a horizontal dashed line. The definition of $\eta(\omega)$ in Eq. (5.6) admits to an interpretation of the characteristic time as an exponential decay constant for $S_{j}(\omega)$ as a function of $j$. Therefore, $\eta(\omega)$ is a convenient average measure of an RTD, with long characteristic times corresponding to large peaks at long residence times. The exponential nature of the decay of the RTD with time should give rise to a Lorentzian component in the power spectral density with half width $f_{1 / 2} \approx[2 \pi R \eta\langle\tau\rangle]^{-1} \approx 1.4 \times 10^{-5} \mathrm{MCSS}^{-1}$, obtained from a numerical evaluation of Eq. (5.11). This low-frequency component should be visible in the spectrum when $f_{1 / 2}<\omega / 2 \pi$.

The result in Eq. (5.11) was obtained in a low-frequency approximation by setting $t_{\mathrm{g}}=0$. Further approximation can be made in the evaluation of Eq. (5.11). The main contribution to the integral in the denominator occurs for $u \approx 1$. Expanding each factor in the integrand in $\epsilon=1-u$ for small $\epsilon$ and substituting $x^{2}=\Xi_{0}(T) \epsilon / H_{0}$ gives the Gaussian approximation,

$$
\begin{aligned}
R \eta & =\left[\frac{\rho_{0}\langle\tau\rangle}{\pi} \sqrt{\frac{2 H_{0}}{\Xi_{0}(T)}} \int_{0}^{\sqrt{\frac{\Xi_{0}(T)}{H_{0}}}} e^{-x^{2}} d x\right]^{-1} \\
& =\left[\frac{\rho_{0}\langle\tau\rangle}{\pi} \sqrt{\frac{\pi H_{0}}{2 \Xi_{0}(T)}} \operatorname{Erf} \sqrt{\frac{\Xi_{0}(T)}{H_{0}}}\right]^{-1}
\end{aligned}
$$

which gives $[2 \pi R \eta\langle\tau\rangle]^{-1}=1.9 \times 10^{-5} \mathrm{MCSS}^{-1}$, and was obtained by inserting quantities from Table $\mathbb{\|}$. The agreement between this value and the data is not as good as that obtained directly from the numerical evaluation of Eq. (5.11). However, the expansion in small $\epsilon$ improves for large $\Xi_{0}(T) / H_{0}$. So this analytic formula for $\eta(\omega)$ would be appropriate for low frequencies and small amplitudes of the external field, i.e. field amplitudes which place the system even deeper into the SD region.

\section{POWER SPECTRAL DENSITIES}

A standard method used to characterize a time series is to calculate its power spectral density (PSD). Figure 0 shows the PSDs of the raw data, short segments of which are shown in Fig. 2. These PSDs are calculated with a standard FFT algorithm implemented using a Welch window [83]. To reduce the variance in the PSDs, each time series is split into several segments. The data is then overlapped in such a way as to obtain an "average" over all the segments for each frequency bin. The details of this method, which we refer to as "smoothing," are given in Ref. [83]. Depending on the number of segments into which the original time series is split, there is a trade-off between frequency resolution and variance reduction. If the time series is split into many segments, the variance per bin is decreased at the expense of lower frequency resolution. Conversely, by partitioning the data into fewer long segments one obtains higher frequency resolution and a smaller low-frequency cutoff, at the expense of a larger variance per bin.

The PSDs for different driving frequencies are shown in Fig. ₹. The spectra in the main part of the plot have been shifted in the vertical direction by arbitrary offsets. The spectra in the inset of Fig. 7 are plotted with no offset. Different amounts of smoothing have been used for the low- and high-frequency regimes. In the low-frequency regime, less smoothing is used. This increases the frequency resolution, enabling one to see sharper peaks in the spectra and sample more of the low-frequency response. In the high-frequency regime, more smoothing is used. Since there is less power in the PSDs for the higher frequencies, a reduction in the variance is a higher priority than frequency resolution. The fourth spectrum shown in Fig. 7, labeled "background," corresponds to thermal fluctuations in a single-phase system. To obtain this spectrum, a simulation was performed on a system with the same size, temperature, and for the same number of MCSS as the other spectra, in a static field of $H_{0} / \sqrt{2}$. The magnetization quickly relaxes to equilibrium. The equilibrium fluctuations are purely thermal, and their time correlations are exponential with a short correlation time of only a few MCSS. The PSD should then have the functional form of a Lorentzian. When plotted on a log-log scale a Lorentzian appears flat at low frequencies, then crosses over into a linear curve with a slope of -2 . The tail on the observed noise background does not appear to have slope $=-2$. However this is most likely due 
to aliasing effects near the Nyquist frequency, $\Omega_{N}=\pi$ [83], which is only about one order of magnitude larger than the inverse correlation time.

To describe the PSD for each frequency, we identify four distinct regions: 1.) the thermal noise region, 2.) the peaks, 3.) the low-frequency region, and 4.) the intermediate region. The inset in Fig. : shows how the PSDs collapse onto the background spectrum in the thermal noise region for large frequencies. The timescale of the thermal fluctuations is much shorter than the shortest period of the external field.

The most prominent features of the PSDs are the sharp peaks. For each driving frequency, the first peak in the spectrum is located at $\omega$, the frequency of the external field. The second peak is located at $3 \omega$, the third peak is located at $5 \omega$, and so on. These odd harmonic peaks arise because the shape of the time series strongly resembles a square wave (refer to Fig. 2). The power $p_{n}$ contained in the $n$th component of the Fourier series for a pure square wave is $p_{n}=16[\sin (n \pi / 2)]^{4} /(n \pi)^{2}$, which is non-zero only for odd $n$ and decays as $n^{-2}$. The zeros in $p_{n}$ for even $n$ survive as zeros in the PSD for a switching process in which the switching probability density $P(t)$ reduces to a delta function [84]. The finite width of $P(t)$ for the present system smoothes out such singularities in the PSD. However, a clear dip in the PSD at twice the driving frequency can be seen for $R=10$. This effect was also noticed by Zhou and Moss in the weak-noise regime for analog simulations of a bistable circuit [85].

The low-frequency region comprises the portion of each spectrum between the first peak and the lowest resolved frequency. The PSD in this region exhibits a strong dependence on the frequency of the external field. For $R=100$, the average intensity in the low-frequency region is approximately constant and is weaker than the fundamental peak intensity by about three orders of magnitude. For $R=2.5$, the slope of the PSD in the low-frequency region is close to -2 over almost two orders of magnitude and contains components comparable in intensity to that of the fundamental peak. This significant amount of power at low frequencies is a consequence of the long residence times, i.e. those residence times longer than the period of the field. The Lorentzian half width predicted from the RTDs in Sec. V VG, $f_{1 / 2} \approx 1.4 \times 10^{-5} \mathrm{MCSS}^{-1}$, is in good agreement with the PSD for $R=2.5$. One can see from Fig. 7 that if the first peak for a PSD is located at a frequency smaller (larger) than approximately $1.4 \times 10^{-5} \mathrm{MCSS}^{-1}$, there are small (large) lowfrequency components. In an analogous fashion, the low-frequency behavior of the PSDs can be deduced by comparing the inverse characteristic time, $[2 \pi R \eta\langle\tau\rangle]^{-1}$, in Fig. 6 with the frequency, $\langle\tau\rangle / R$ (in units of $\mathrm{MCSS}^{-1}$ ), which is shown as a short-dashed line in the same figure. For low driving frequencies, $\langle\tau\rangle / R<[2 \pi R \eta\langle\tau\rangle]^{-1}$. Therefore, most of the residence times are less than a period of the external field, which corresponds to small low-frequency components in

the PSDs. For high driving frequencies, $\langle\tau\rangle / R>[2 \pi R \eta\langle\tau\rangle]^{-1}$, and large low-frequency components appear in the PSDs.

The intermediate region is the portion of each spectrum between the highest-order visible peak and the thermal-noise region. This region is discussed last because it is best understood as a crossover from the peaks to the thermal-noise region. The structure of the odd-harmonic peaks is easiest to discern for $R=100$, where the first five peaks are clearly visible. The higher-frequency harmonics cannot be seen for two reasons: the peak positions become very closely spaced on a logarithmic scale, and the intensity of the peaks becomes too small to be seen above the fluctuations in the PSD. So the intermediate region may be thought of as an envelope of the high-frequency, odd-harmonic peaks. A log-log plot of $p_{n}$ vs. odd $n$ yields a line with a slope of -2 . However, the portion of the PSD just to the left of the thermal-noise region is decreasing with a slope steeper than -2 . This sharp falloff is a consequence of the finite growth time of a critical droplet. A finite growth time effectively "smoothes out" the sharp corners of the square-wave response of the magnetization, which introduces a cutoff for the highest-frequency components of $p_{n}$ in the intermediate region.

\section{HYSTERESIS LOOPS, CORRELATION, AND PERIOD-AVERAGED MAGNETIZATION}

To characterize the behavior of an entire time series we calculate the following integrals of the magnetization,

$$
\begin{aligned}
A & =-\oint m(H) d H \\
B & =\frac{\omega}{2 \pi} \oint m(t) H(t) d t \\
Q & =\frac{\omega}{2 \pi} \oint m(t) d t,
\end{aligned}
$$

where $A$ is the hysteresis-loop area, $B$ is the correlation between the external field and the system magnetization, and $Q$ is the period-averaged magnetization. These quantities are calculated over each period in the entire time series. From the resulting "filtered" time series we construct histograms to obtain the probability densities of $A, B$, and $Q$ for each separate frequency of the external field. 
In Sec. VII A we compare our MC data and theoretical calculations of $A$ and $B$. In particular, we comment on the low-frequency power-law scaling for $A$ which has been put forth in numerous studies. At the end of this section we show our results for $B$ and identify $A$ and $B$ as components of a nonlinear response function. In Sec. VIIB we discuss $Q$ and the absence of a dynamic phase transition in the SD region for this system.

\section{A. Hysteresis loops and correlation}

The hysteresis-loop area, $A$, represents the energy dissipated during a single period of the applied field. It is therefore one of the most important physical quantities characterizing hysteretic systems, and it is frequently measured in experiments. Under the conditions of stochastic magnetization reversal, $A$ and $B$ are random variables with nontrivial statistical properties. Figure 8 shows the probability densities of $A$. For all values of $R \lesssim 10$, there is a sharp peak near zero. This peak is denoted as $A_{0}$ and corresponds to the field cycles during which $m(t)$ does not switch sign, but merely fluctuates near $\pm m_{\text {eq }}$. The second peak, $A_{1}$, is located near $A /\left(4 H_{0}\right)=0.5$. It represents field cycles during which $m(t)$ switches sign once. The third peak, $A_{2}$, located near a loop area of $A /\left(4 H_{0}\right)=1.0$, represents cases when $m(t)$ switches sign twice within the same period. When the period of the field increases, the weight in the peaks moves from low to high values of $A$. For $H(t)$ with longer periods, the magnetization has a higher probability of switching once or twice during a single period, thus transferring more weight to the peaks $A_{1}$ and $A_{2}$. For very low frequencies, the magnetization almost always switches twice in each period, giving loops of type $A_{2}$. At the same time, switching occurs earlier in each half-period. This reduces the average loop area, giving rise to the type of pdf shown for $R=100$ in Fig. 8. Figure 9 shows typical hysteresis loops with values of $A$ corresponding to the three peaks, $A_{0}, A_{1}$, and $A_{2}$.

The fraction of events contained in each of these peaks we denote as $f_{0}, f_{1}$, and $f_{2}$. These are the probabilities that the magnetization switches zero, one, or two times in a full period of the field. They depend on the probability that the sign of $m(t)$ is opposite that of $H(t)$ immediately after $H(t)$ has switched sign at the beginning of the period. We call this phase probability $p_{-}$. In terms of $p_{-}$and $P_{\text {not }}(\omega)$ we have:

$$
\begin{aligned}
& f_{0}=P_{\text {not }}(\omega) \\
& f_{1}=p_{-} P_{\text {not }}(\omega)\left(1-P_{\text {not }}(\omega)\right)+\left(1-p_{-}\right)\left(1-P_{\text {not }}(\omega)\right) \\
& f_{2}=p_{-}\left(1-P_{\text {not }}(\omega)\right)^{2} .
\end{aligned}
$$

The phase whose probability is given by $p_{-}$changes in a two-state Markov process described by a transition matrix fully determined by $P_{\text {not }}(\omega)$. It is easy to show that the stationary value of $p_{-}=1 /\left(1+P_{\text {not }}(\omega)\right)$ [86]. After the phase distribution has reached its stationary state, we therefore get

$$
\begin{aligned}
& f_{0}=P_{\mathrm{not}}(\omega) \\
& f_{1}=\frac{2 P_{\mathrm{not}}(\omega)\left(1-P_{\mathrm{not}}(\omega)\right)}{\left(1+P_{\mathrm{not}}(\omega)\right)} \\
& f_{2}=\frac{\left(1-P_{\mathrm{not}}(\omega)\right)^{2}}{\left(1+P_{\mathrm{not}}(\omega)\right)} .
\end{aligned}
$$

These expressions satisfy the normalization condition $f_{0}+f_{1}+f_{2}=1$. The $f_{i}$ are directly related to the RTD peak strengths, $S_{j}$, through their common dependence on $P_{\text {not }}$. In particular, $f_{0}=P_{\text {not }}=1-S_{1}$.

It is less clear how to separate the peaks when measuring the fractions from the MC data. For example, with cutoffs at 0.333 and 0.666 an event is considered part of peak $A_{0}$ if $0<A / 4 H_{0}<0.333$, part of peak $A_{1}$ if $0.333<A / 4 H_{0}<$ 0.666 , and part of peak $A_{2}$ if $0.666<A / 4 H_{0}<1.0$. Different cutoffs were tried and judged by how well they fit the theoretical results. No particular choice produced a fit that was qualitatively better than others. Comparisons of the $f_{i}$ calculated from theory and simulation are shown in Fig. 10. The data points are measured from the loop-area distributions, using cutoffs at 0.333 and 0.666. The solid curves are calculated from Eqs. (7.5a-c), using the theoretical values for $P_{\text {not }}(\omega)$. Considering that no free parameters are used in the theory, it agrees well with the simulation data.

The average loop area for a specific frequency is a quantity often displayed in experimental and numerical studies of hysteresis. It can also be obtained in the present case, and we do so below. However, for stochastic hysteresis this is not a very useful quantity at higher frequencies, where the distributions are trimodal. The means of the distributions in Fig. 8 are shown in Fig. 11. Note that the vertical bars are not error bars, but rather give the standard deviations of the distributions. The dotted curve is obtained by assuming that the fluctuations of the loop areas are small in each of the three peaks. An approximate value for the scaled loop area can be assigned to each of the three peaks, $\left\langle A_{0}\right\rangle / 4 H_{0} \approx 0.00,\left\langle A_{1}\right\rangle / 4 H_{0} \approx m_{\mathrm{eq}} / 2$, and $\left\langle A_{2}\right\rangle / 4 H_{0} \approx m_{\mathrm{eq}}$. With these choices we assume that the magnetization switches when the absolute value of the external field is close to a maximum. The average loop area is calculated by weighting these values by the fraction of events in each peak, 


$$
\frac{\langle A\rangle_{\mathrm{HF}}}{4 H_{0}}=f_{0}(\omega)\left(\frac{\left\langle A_{0}\right\rangle}{4 H_{0}}\right)+f_{1}(\omega)\left(\frac{\left\langle A_{1}\right\rangle}{4 H_{0}}\right)+f_{2}(\omega)\left(\frac{\left\langle A_{2}\right\rangle}{4 H_{0}}\right)
$$

where "HF" stands for "high-frequency." This expression breaks down in the low-frequency limit, as $f_{2} \rightarrow 1$ and $\left\langle A_{2}\right\rangle$ becomes strongly frequency dependent. Next we discuss this effect.

For any finite time series there is a sufficiently low frequency such that the magnetization always switches during every half-period of the field. For very low frequencies the magnetization switches, on average, before the field reaches its extreme value during every half-period. Therefore, the loop-area distribution becomes unimodal, and the mean loop area decreases with decreasing frequency. Since the probability density of switching times, $P(t)$, is narrow and unimodal for low frequencies (refer to Fig. 3), we can choose the typical time when the magnetization switches during each half period, $t_{\mathrm{s}}$, to be either its mean, mode, or median. We use the latter because it is analytically more tractable and has an analytic asymptotic approximation. To determine the median of $P(t)$ we must solve $F\left(t_{\mathrm{s}}\right)=1 / 2$, where the cumulative probability distribution $F(t)$ is given by Eq. (4.6). To simplify the form of this equation we note that for low frequencies the hysteresis loops are square (refer to Fig. $9(\mathrm{~d})$ ), which means that the growth time becomes small compared to $t_{\mathrm{s}}$ and can be ignored. Thus, the equation for $t_{\mathrm{s}}$ becomes

$$
F\left(t_{\mathrm{s}}\right)=1-\exp \left[-\int_{0}^{t_{\mathrm{s}}} \rho\left(t^{\prime}\right) d t^{\prime}\right]=\frac{1}{2} .
$$

After rearranging and substituting $x=\Xi_{0} /\left[H_{0} \sin (\omega t)\right]^{d-1}$, we obtain

$$
\begin{aligned}
\ln 2 & =\int_{0}^{t_{\mathrm{s}}} \rho\left(t^{\prime}\right) d t^{\prime} \\
& =\rho_{0} \frac{e^{\frac{\Xi_{0}(T)}{H_{0}^{d-1}}}}{H_{0}^{K}} \int_{0}^{t_{\mathrm{s}}}\left[H_{0} \sin \left(\omega t^{\prime}\right)\right]^{K} e^{-\frac{\Xi_{0}(T)}{\left[H_{0} \sin \left(\omega t^{\prime}\right)\right]^{d-1}} d t^{\prime}} \\
& =\rho_{0} \frac{e^{\frac{\Xi_{0}(T)}{H_{0}^{d-1}}} \Xi_{0}^{\frac{K+1}{d-1}}}{H_{0}^{K+1}(d-1) \omega} \int_{\frac{\Xi_{0}}{H_{\mathrm{s}}^{d-1}}}^{\infty} \frac{x^{-\frac{K+d}{d-1}} e^{-x}}{\sqrt{1-\left[\frac{1}{H_{0}}\left(\frac{\Xi_{0}}{x}\right)^{\frac{1}{d-1}}\right]^{2}}} d x .
\end{aligned}
$$

As the frequency is decreased the switching field, $H_{\mathrm{s}}=H_{0} \sin \left(\omega t_{\mathrm{s}}\right)$, decreases. For very low frequencies $x \gg \Xi_{0} / H_{0}^{d-1}$ and the radical in Eq. (7.10) is of order one, resulting in the following expression

$$
\ln 2=\rho_{0} \frac{e^{\frac{\Xi_{0}(T)}{H_{0}^{d-1}}} \Xi_{0}^{\frac{K+1}{d-1}}}{H_{0}^{K+1}(d-1) \omega} \int_{\frac{\Xi_{0}}{H_{\mathrm{s}}^{d-1}}}^{\infty} x^{-\frac{K+d}{d-1}} e^{-x} d x
$$

which may also be derived through a linear approximation to the sinusoidal field, $H(t) \approx H_{0} \omega t$. After rearranging and simplifying this becomes [50]

$$
C H_{0} \omega=\Gamma\left(1-\frac{K+d}{d-1}, \frac{\Xi_{0}}{H_{\mathrm{s}}^{d-1}}\right)
$$

where $\Gamma(a, x)$ is the incomplete gamma function [87], and we define

$$
C=\frac{\ln 2 H_{0}^{K}(d-1) e^{-\frac{\Xi_{0}(T)}{H_{0}^{d-1}}} \Xi_{0}^{-\frac{K+1}{d-1}}}{\rho_{0}} .
$$

With $d=2, K=3$, and the values found in Table 1 , $C=0.101 J^{-1}$ MCSS. For small $\omega$ the hysteresis loops are practically square, so the scaled loop area in the low-frequency (LF) limit can be expressed as

$$
\frac{\langle A\rangle_{\mathrm{LF}}}{4 H_{0}} \approx m_{\mathrm{eq}} \frac{H_{\mathrm{S}}(\omega)}{H_{0}}
$$

The switching field $H_{\mathrm{S}}(\omega)$ is obtained from a numerical solution of Eq. (7.10), and the result for $\langle A\rangle_{\mathrm{LF}} / 4 H_{0}$ is shown as the solid curve in Fig. 11(a)-(b). Figure 11(b) shows the good agreement between this parameter-free calculation and the MC data for frequencies $1 / R \leq 0.05$. 
One can obtain an approximate analytic solution by taking the first term in the asymptotic expansion 87

$$
\Gamma(a, x) \sim x^{a-1} e^{-x}\left[1+\frac{a-1}{x}+\ldots\right]
$$

for large $x$. By ignoring the factor $x^{a-1}$, one obtains a completely analytic solution for $H_{\mathrm{s}}$ in the extreme LF limit, resulting in the asymptotic, logarithmic frequency dependence for the loop area

$$
\langle A\rangle_{\mathrm{LF}} \approx 4 \Xi_{0}^{\frac{1}{d-1}}\left[-\ln \left(C H_{0} \omega\right)\right]^{-\frac{1}{d-1}} .
$$

In Fig. 11 we show calculations of both this asymptotic analytic result (short-dashed curve) for the loop area and the loop area obtained from the numerical solution (solid curve) of Eq. (7.10). The dashed lines in Fig. 11(b) are linear least-squares fits to the full numerical solution over almost four decades in frequency. The slopes of these fit lines would give an "effective" exponent b for the loop area, $A \propto \omega^{b}$. For the frequency regime shown, these effective power-law exponents for the loop area depend on frequency. These results show no evidence of an overall power-law relationship between the frequency and the loop area.

To the best of our knowledge, the present study is the first to give a complete solution of $A$ and emphasize its possible significance for the low-frequency power-law behavior of $A$ reported in the literature. This issue is discussed in further detail in a separate paper [50|. Theoretical considerations of nucleation effects on hysteresis have been mentioned previously in the literature [6,26, 62], reporting the same asymptotic frequency behavior for the loop area as in Eq. (7.16). However, we stress that the purely logarithmic dependence of the loop area on frequency and amplitude of the external field will approach the exact solution obtained from Eq. (7.12) only for extremely low frequencies, as illustrated by the poor agreement between the short-dashed and solid curves in Fig. 11(b). The units of frequency used in our simulations may be converted roughly into Hertz by equating a phonon frequency with an inverse Monte Carlo step per spin, $\bar{\nu}=1 \mathrm{MCSS}^{-1}$, with $\bar{\nu}=10^{9}-10^{12} \mathrm{~Hz}$ as a reasonable estimate. Then, the lowest frequency calculated for the solid curve in Fig. 11(b) corresponds to a field period on the order of thousands to millions of years. The full asymptotic behavior of the loop area is realized for frequencies lower still, and is therefore well outside the range of feasible experiments. However, the lowest-frequency MC data point corresponds to a field period on the order of microseconds to seconds, suggesting the low-frequency SD behavior of the loop area described by our full numerical calculation could be experimentally observable.

In extensive simulations, Acharyya and Chakrabarti have studied the hysteretic response of Ising systems with respect to changes in field amplitude and frequency, temperature, system size, and system dimension [52]. In particular, they have presented power-law scaling relations for $A$. However, their simulations in $d=2$ are all done with very large field amplitudes. For the temperatures used in their papers, these field amplitudes place their system in a regime we refer to as the "strong-field (SF) region," where the size of a critical droplet becomes comparable to the lattice constant $a$, and the droplet picture of metastable decay breaks down [45]. The investigation of hysteresis in kinetic Ising models by Lo and Pelcovits [57] is not restricted to the SF region. In particular, the range of field amplitudes used places their simulations in the SD, MD and SF regions as the amplitude is increased. The hysteretic response, including the loop area, requires qualitatively different theoretical descriptions in each of these regions. This drastic change in the Ising model contrasts with a coherent rotation model of magnetization reversal, for which increasing the field strength would merely decrease the energy barrier that the system must overcome for the magnetization to be aligned with the field. For the kinetic Ising model and other spatially extended systems however, increasing the field not only decreases the free-energy barrier for forming a single critical droplet of the stable phase, it also changes the reversal mode. Our results emphasize the importance of a knowledge of the decay mode in order to obtain the correct frequency dependence of the average loop area over a wide range of frequencies.

Mahato and collaborators [88.89 also considered a driven bistable system in the presence of noise. From the RTDs they calculate what they describe as an "average hysteresis loop," and they propose a maximum in this quantity with respect to noise strength as a manifestation of SR. We find their definition of a loop area rather unphysical, except for very low frequencies. However, the role of the hysteresis-loop area as a measure of energy dissipation indicates that a maximum in $A$ may be considered an aspect of SR, which we suggest could be termed "stochastic energy resonance."

Relatively few studies have considered $B$, the correlation of the magnetization with the external field. For Ising models subject to oscillating fields, these studies [40,41] have found a temperature at which the correlation attains a maximum value for a given frequency, amplitude, and system size. However, as recently pointed out by Acharyya [90], this feature is not a sign of SR. These simulations are mostly performed above $T_{c}$ in a regime where the metastable state no longer exists. Therefore, comparison of our simulations or analytic results with these studies is not relevant.

Our theoretical derivations of $B$ are similar to those for $A$ and are also given as high- and low-frequency approximations. Figure 12 shows our MC data for $B$ along with the theoretical result for the high-frequency regime (dotted curve) and the low-frequency regime (solid curve). We assume that $H_{\mathrm{s}} \approx H_{0}$ for high frequencies, and that the 
magnetization switching is stochastic with either zero, one, or two switches every period. The only non-zero contribution to the correlation comes from those periods when the magnetization switches only once. We use notation analogous to the high-frequency loop-area calculation for each of these three contributions, $\left\langle B_{0}\right\rangle /\left(2 H_{0} / \pi\right) \approx 0.0$, $\left\langle B_{1}\right\rangle /\left(2 H_{0} / \pi\right) \approx-m_{\mathrm{eq}} / 2$, and $\left\langle B_{2}\right\rangle /\left(2 H_{0} / \pi\right) \approx 0.0$, with $\langle B\rangle_{\mathrm{HF}} /\left(2 H_{0} / \pi\right)$ calculated as a weighted average similar to Eq. (7.6),

$$
\frac{\langle B\rangle_{\mathrm{HF}}}{2 H_{0} / \pi}=f_{0}(\omega)\left(\frac{\left\langle B_{0}\right\rangle}{2 H_{0} / \pi}\right)+f_{1}(\omega)\left(\frac{\left\langle B_{1}\right\rangle}{2 H_{0} / \pi}\right)+f_{2}(\omega)\left(\frac{\left\langle B_{2}\right\rangle}{2 H_{0} / \pi}\right) .
$$

Figure 12 shows that $\langle B\rangle_{\mathrm{HF}} /\left(2 H_{0} / \pi\right) \rightarrow 0$ as $\omega \rightarrow 0$ and as $\omega \rightarrow \infty$, which is a direct consequence of the behavior of $f_{1}(\omega)$ (see Fig. $\left.10(\mathrm{~b})\right)$.

The low-frequency calculation for $B$ is also similar to that for $A$ and assumes that the magnetization switches abruptly at $\left|H_{\mathrm{s}}\right|<H_{0}$ during each half-period. This assumption together with the definition of $B$, Eq. (7.2), yields

$$
\frac{\langle B\rangle_{\mathrm{LF}}}{2 H_{0} / \pi}=m_{\mathrm{eq}} \sqrt{1-\left(\frac{H_{\mathrm{s}}(\omega)}{H_{0}}\right)^{2}} .
$$

As for $A$, the switching field $H_{\mathrm{s}}(\omega)$ is obtained from a numerical solution of Eq. (7.10). There is good agreement with the MC data for the two lowest frequencies. As the frequency increases the theoretical result approaches zero. However, the assumption that the magnetization switches during every half-period begins to break down around $1 / R=0.05$, where the MC result for $B$ passes through zero.

Although the system studied here is both stochastic and highly nonlinear, the physical significance of the integrals $A$ and $B$ can be clarified by comparison with deterministic linear response theory. In that limit one easily finds that $A /\left(\pi H_{0}^{2}\right)$ and $2 B / H_{0}^{2}$ correspond to the dissipative and reactive parts of the complex linear response function, respectively. It is therefore natural to combine $A$ and $B$ into an analogous nonlinear response function,

$$
X\left(H_{0}, T, \omega\right)=\frac{1}{H_{0}^{2}}\left[2 B+\frac{i}{\pi} A\right] .
$$

The maximum in $A$ and the sign change in $B$, which occur close together in frequency, are characteristic behaviors of the dissipative and a reactive parts of a response function near resonance. It is reasonable to associate this behavior with SR. However, as we pointed out at the end of Sec. VB, $m(t)$ remains essentially synchronized with $H(t)$ as the driving frequency is lowered further below this narrow frequency range. The system then switches reliably during every half-period of the field, while the switching occurs earlier and earlier in the half-period as the frequency is lowered. In this low-frequency regime, the norm of $X$ remains close to its maximum value of $4 m_{\mathrm{eq}} /\left(H_{0} \pi\right)$, and its phase gives a meaningful measure of the period-averaged phase lag. The latter increases monotonically from zero at $\omega=0$ to $\pi / 2$ at the frequency where $B$ crosses zero. We believe the system should be considered as resonant in this whole range of low frequencies.

\section{B. Period-Averaged Magnetization}

The period-averaged magnetization $Q$ has been considered as a "dynamic order parameter" for systems exhibiting hysteresis 32,52 57]. Those studies of the Ising model have suggested the existence of a dynamic phase transition between $\langle Q\rangle \neq 0$ and $\langle Q\rangle=0$. As for the hysteresis-loop areas, the statistical properties of the period-averaged magnetization in the SD region are not well characterized simply by its mean. Figure 13 shows the probability densities of $Q$ in the SD region. For all but the very highest values of $R$, the distributions show two sharp peaks near $Q= \pm m_{\mathrm{eq}}$ due to $m(t)$ oscillating near the spontaneous magnetization during most of the field cycles. The contributions to the $Q$ distributions near $Q=0$ occur when the magnetization switches twice in one period. The contributions to the peak centered around $Q=+0.5$ occur for those periods in which the magnetization switches only once. Note that there is not a corresponding peak at $Q=-0.5$. This is an effect of the way we calculate the period-averaged magnetization, which considers the beginning of a period to start when $H(t)=0$ and $\dot{H}>0$. We would have obtained a peak near $Q=-0.5$ if we had started with $H(t)=0$ and $\dot{H}<0$.

Even by inspecting the distributions for $Q$, no dynamic phase transition can be seen. While the means of the distributions for high (low) frequencies are nonzero (zero), this happens smoothly as weight shifts from the peaks near $Q= \pm 1$ to the peak at $Q=0$. As we will show in a forthcoming paper, the situation is quite different in the MD region, where we find strong evidence for a dynamic phase transition [51]. 


\section{DISCUSSION}

The mechanism by which a metastable phase decays depends sensitively on the system size, the temperature, and the strength of the applied field. For small systems and weak fields, the decay proceeds through the nucleation and growth of a single droplet of overturned spins. This regime has been termed the single-droplet (SD) region. In this region the magnetization response consists of rapid transitions between two states; one with the majority of the spins up, and one with the majority of the spins down. The resulting time series is well described in terms of a Poisson process with a time-dependent rate obtained from the nucleation rate and growth velocity of droplets of the stable phase. The time dependence enters the nucleation rate by replacing the constant field $H$ by $H(t)=H_{0} \sin (\omega t)$. This central idea provides the analytic framework for theoretical descriptions of the quantities measured from our MC simulations. These quantities include residence-time distributions (RTDs), power spectral densities (PSDs), hysteresis-loop areas, and the correlation between the magnetization and the oscillating field. The agreement between all of our theoretical calculations and the MC data is very good, especially considering that the theory contains no adjustable parameters. All of the constants used are either known from droplet theory or are measured from MC simulations of field reversal in kinetic Ising models. To the best of our knowledge, the present study is the first which explicitly considers hysteresis for the Ising model in the SD regime.

The frequency dependence of the RTD peak shapes and peak strengths are calculated by numerically evaluating analytic expressions obtained from a time-dependent extension of classical nucleation theory. The good agreement between our theoretical calculations and MC data supports the model of magnetization switching as a Poisson process with a variable rate, given by substituting a sinusoidal field dependence for the static field in the nucleation rate.

The frequency dependence of the RTD peak strengths, the hysteresis-loop areas, and the correlation between the magnetization and the field all indicate the presence of stochastic resonance (SR) in the two-dimensional Ising model in this parameter regime. This observation is consistent with recent studies of SR in other systems of coupled bistable elements, some of which pointed out the importance of nucleation of kink-antikink pairs to what has been termed array enhanced stochastic resonance (AESR). For any nucleation process, there should be crossovers between coexistence (CE), single-droplet (SD), and multidroplet (MD) types of behavior as a consequence of the interplay between the sizes and separations of the critical fluctuation(s) and the size of the system. We believe these crossovers should be relevant to the dependence of the amount of enhancement on the number of elements observed in other systems exhibiting AESR as well.

We also calculate the hysteresis-loop area, $A$, in the low- and high-frequency regimes. Because of its role as a measure of the energy dissipation in the system, this is a quantity of particular experimental significance. For high frequencies the loop-area distributions are trimodal due to the stochastic switching behavior. In this regime we calculate $\langle A\rangle$ as a weighted average of the loop areas obtained when the magnetization switches zero, one, or two time(s) during a period of the field. For the low-frequency regime we obtain an analytic expression for $\langle A\rangle$. Our theoretical calculation agrees well with our MC results and predicts an extremely slow crossover to a logarithmic dependence of the loop area on $H_{0} \omega$. The switching dynamics is dominated by nucleation and indicates no overall power-law dependence for the loop area on field amplitude and/or frequency, in contrast to what has been claimed in other simulational and experimental studies. However, we emphasize that numerical analysis of data generated by our analytic solution, even over several frequency decades, could easily lead to the conclusion that the data were taken from a power law.

The period-averaged magnetization, $Q$, has been proposed as an "order parameter" associated with a dynamic phase transition in kinetic Ising models. However, in the parameter range studied here, the probability densities of $Q$ show no sign of a sharp transition as the frequency of the external field is varied. Indeed, due to the multi-peaked nature of the distributions for intermediate frequencies, the mean value of $Q$ is not a useful quantity in the SD region. In the MD region the behavior of $Q$ is radically different, as we will discuss in forthcoming papers [51].

We also computed the power spectral densities (PSDs) from the simulated magnetization time series. We qualitatively explain the various features of the spectra in the full frequency range from the lowest observable frequencies to the rapid fluctuations due to thermal noise. Specifically, we make a connection between the RTDs and the lowfrequency behavior in the PSDs through the characteristic time of the RTDs. Inverse characteristic times that are smaller (larger) than the frequency of the applied field correspond to large (small) low-frequency components in the PSDs. Our theoretical derivation of the characteristic time also agrees well with the characteristic times obtained from the simulated RTDs, except at very high frequencies. The relatively poor agreement between the MC data and the theory for high frequencies of the external forcing field is common to most of the quantities measured and is most likely due to the poor quality of the MC data for high frequencies. However, it could also be a sign of breakdown in the adiabatic approximation underlying the assumption that the functional form of the nucleation rate and the calculation of the growth-time corrections do not change for high frequencies $[79]$.

In summary, we have studied stochastic hysteresis in the kinetic Ising model, a spatially extended, bistable system 
with thermal fluctuations. We emphasize not only the detailed differences between hysteresis in mean-field models and Ising models, but also the qualitatively different response that the Ising model displays for particular regimes of system size and field amplitude. Our theoretical and numerical study is the first to consider the effects of these different decay regimes on hysteresis, which may be relevant to the interpretation of simulational and experimental results. Especially for certain technological applications, an Ising system should be a good candidate to model the behavior of ferromagnetic and ferroelectric materials in oscillating external fields. Finally we note that the quantities that we have analyzed numerically could all be measured in experiments on hysteresis in a variety of systems and analyzed by methods essentially identical to our analysis of the MC data.

\section{ACKNOWLEDGMENTS}

We would like to thank P.D Beale, G. Brown, M. Grant, W. Klein, M. Kolesik, R.A. Ramos, and H. Tomita for useful discussions. S.W.S and P.A.R appreciate hospitality and support from the Colorado Center for Chaos and Complexity during the 1997 Workshop on Nucleation Theory and Phase Transitions. Supported in part by the FSU Center for Materials Research and Technology (MARTECH), by the FSU Supercomputer Computations Research Institute (SCRI) under DOE Contract No. DE-FC05-85ER25000, and by NSF Grants No. DMR-9315969, DMR-9634873, and DMR-9520325.

\section{APPENDIX A: DERIVATION OF THE RESIDENCE-TIME DISTRIBUTIONS}

The $\alpha^{\text {th }}$ residence time is defined as

$$
\Delta^{\alpha}=t_{\uparrow}^{\alpha}+\theta^{\alpha},
$$

where the times $t_{\uparrow}^{\alpha}$ and $\theta^{\alpha}$ are shown schematically in Fig. 14. We define $t_{\uparrow}$ as the time when a switching event takes place, as measured from the first time at which $H(t)=0$, after the previous switching event. Without loss of generality, this time can be set to $t=0$. $\theta$ is the time from a switching event to the next change in the sign of the external field. This decomposition of the residence time facilitates the calculation of the probability density functions (pdf) for both $t_{\uparrow}^{\alpha}$ and $\theta^{\alpha}$. When $t_{\uparrow}$ falls during the $j=1$ period (see Fig 14 for an explanation of the indexing scheme), its probability density is given by Eq. (4.14), i.e. $p_{\uparrow}\left(t_{\uparrow}\right)=P\left(t_{\uparrow}\right)$. We easily generalize to the case when $t_{\uparrow}$ falls during the $j$ th period. This is obtained by finding the probability that, given the magnetization has not switched in the previous $j-1$ periods, it switches at a time $t_{\uparrow}$ during the $j$ th period,

$$
p_{\uparrow}\left(t_{\uparrow}\right)=\left[P_{\mathrm{not}}(\omega)\right]^{j-1} P\left[t_{\uparrow}-(j-1) \frac{2 \pi}{\omega}\right] .
$$

The pdf for $\theta, p_{\theta}(\theta)$, is calculated from $p_{\uparrow}\left(t_{\uparrow}\right)$ by using the fact that $\theta^{\alpha}$ and $\theta^{\alpha+1}$ should be independent and identically distributed. Then the following substitution holds,

$$
t_{\uparrow}^{\alpha}+\theta^{\alpha+1}=\left(j-\frac{1}{2}\right) \frac{2 \pi}{\omega},
$$

which gives

$$
\begin{aligned}
p_{\theta}\left(\theta^{\alpha}\right) & =p_{\theta}\left(\theta^{\alpha+1}\right) \\
& =\sum_{j=1}^{\infty} p_{\uparrow}\left[\left(j-\frac{1}{2}\right) \frac{2 \pi}{\omega}-\theta^{\alpha+1}\right] \\
& =\sum_{j=1}^{\infty}\left[P_{\mathrm{not}}(\omega)\right]^{j-1} P\left[\left(j-\frac{1}{2}\right) \frac{2 \pi}{\omega}-\theta^{\alpha}-(j-1) \frac{2 \pi}{\omega}\right] \\
& =P\left(\frac{\pi}{\omega}-\theta^{\alpha}\right) \sum_{j=1}^{\infty} P_{\mathrm{not}}(\omega)^{j-1} .
\end{aligned}
$$

Thus, 


$$
p_{\theta}(\theta)=P\left(\frac{\pi}{\omega}-\theta\right)\left[1-P_{\text {not }}(\omega)\right]^{-1}
$$

The pdf of the total residence time, $\Delta=t_{\uparrow}+\theta$, is given by the convolution of the pdfs of each term:

$$
\begin{aligned}
\Pi(\Delta) & =\int_{\theta_{a}(\Delta)}^{\theta_{b}(\Delta)} p_{\uparrow}(\Delta-\theta) p_{\theta}(\theta) d \theta \\
& =\frac{\left[P_{\mathrm{not}}(\omega)\right]^{j-1}}{\left[1-P_{\mathrm{not}}(\omega)\right]} \int_{\theta_{a}(\Delta)}^{\theta_{b}(\Delta)} P\left[\Delta-\theta-(j-1) \frac{2 \pi}{\omega}\right] P\left(\frac{\pi}{\omega}-\theta\right) d \theta,
\end{aligned}
$$

where $j=\lceil\omega \Delta /(2 \pi)\rceil$. The notation $\lceil x\rceil$ is defined as the smallest integer greater than $x$. For $j=1$, the integration limits are given by

$$
\begin{aligned}
\theta_{a}(\Delta) & =\operatorname{Max}\left[0, \Delta-\frac{\pi}{\omega}\right] \\
\theta_{b}(\Delta) & =\operatorname{Max}\left[0, \operatorname{Min}\left[\Delta-t_{0}, \frac{\pi}{\omega}-t_{0}\right]\right],
\end{aligned}
$$

which ensure that the integrand in the equation for $\Pi(\Delta)$ is positive. To implement the calculation of the RTDs, $\Pi(\Delta)$ is numerically integrated for $j=1$ at 50 equally spaced values in the interval $0<\Delta<2 \pi / \omega$, to generate the first peak in the RTD. To obtain the second peak, the values of the distribution are shifted to the next interval $2 \pi / \omega<\Delta<4 \pi / \omega$, and reduced by a factor of $P_{\text {not }}(\omega)$. This process can be repeated to obtain all of the higher-order peaks in the RTD.

a) Present address at Florida State University E-mail: sides@scri.fsu.edu

b) Present and permanent address at Florida State University E-mail: rikvold@scri.fsu.edu

c) Present address at Florida State University E-mail: novotny@scri.fsu.edu

[1] C. P. Steinmetz, Trans. Am. Inst. Electr. Eng. 9, 3 (1892).

[2] I. D. Mayergoyz, Mathematical Models of Hysteresis (Springer, New York, 1991).

[3] A. Aharoni, Introduction to the Theory of Ferromagnetism (Clarendon, Oxford, 1996).

[4] Y. Ishibashi and Y. Takagi, J. Phys. Soc. Jpn. 31, 506 (1971).

[5] H. Orihara and Y. Ishibashi, J. Phys. Soc. Jpn. 61, 1919 (1992).

[6] P. D. Beale, Integrated Ferroelectrics 4, 107 (1994).

[7] M. Rao and R. Pandit, Phys. Rev. B 43, 3373 (1991).

[8] A. J. Bard and L. R. Faulkner, Electrochemical Methods: Fundamentals and Applications (Wiley, New York, 1980).

[9] P. A. Rikvold et al., Surf. Sci. 335, 389 (1995).

[10] A. Cheng and M. Chaffrey, J. Phys. Chem. 100, 5608 (1996).

[11] A. H. Nayfeh and B. Balachandran, Applied Nonlinear Dynamics (Wiley, New York, 1995).

[12] A. Visintin, Differential Models of Hysteresis (Springer, Berlin, 1994).

[13] M. Brokate and J. Sprekels, Hysteresis and Phase Transitions (Springer, New York, 1996).

[14] Y. Martin and H. Wickramasinghe, Appl. Phys. Lett. 50, 1455 (1987).

[15] T. Chang, J. G. Zhu, and J. Judy, J. Appl. Phys. 73, 6716 (1993).

[16] M. Lederman, G. Gibson, and S. Schultz, J. Appl. Phys. 73, 6961 (1993).

[17] M. Lederman, D. Fredkin, R. O'Barr, and S. Schultz, J. Appl. Phys. 75, 6217 (1994).

[18] M. Lederman, S. Schultz, and M. Ozaki, Phys. Rev. Lett. 73, 1986 (1994).

[19] H. L. Richards, S. W. Sides, M. A. Novotny, and P. A. Rikvold, J. Magn. Magn. Mater. 150, 37 (1995).

[20] H. L. Richards, M. A. Novotny, and P. A. Rikvold, Phys. Rev. B 54, 4113 (1996).

[21] H. L. Richards et al., Phys. Rev. B 55, 11521 (1997).

[22] M. Kolesik, M. A. Novotny, and P. A. Rikvold, Phys. Rev. B. 56, 11791 (1997).

[23] M. Kolesik, M. A. Novotny, and P. A. Rikvold, Mater. Res. Soc. Conf. Proc. Ser. (1997).

[24] Y. He and G. Wang, Phys. Rev. Lett. 70, 2336 (1993).

[25] M. Rao, H. Krishnamurthy, and R. Pandit, J. Phys. C 1, 9061 (1989).

[26] M. Rao, H. Krishnamurthy, and R. Pandit, Phys. Rev. B 42, 856 (1990).

[27] M. Rao, H. Krishnamurthy, and R. Pandit, J. Appl. Phys. 67, 5451 (1990).

[28] Q. Jiang, H.-N. Yang, and G.-C. Wang, Phys. Rev. B 52, 14911 (1995). 
[29] R. Benzi, A. Sutera, and A. Vulpiani, J. Phys. A 14, L453 (1981).

[30] A. R. Bulsara and L. Gammaitoni, Physics Today 49, Issue 3, 39 (1996).

[31] P. Jung, G. Gray, and R. Roy, Phys. Rev. Lett. 65, (1990).

[32] T. Tomé and M. J. de Oliveira, Phys. Rev. A 41, 4251 (1990).

[33] C. Luse and A. Zangwill, Phys. Rev. E 50, 224 (1994).

[34] M. Löcher, G. Johnson, and E. Hunt, Phys. Rev. Lett. 77, 4698 (1996).

[35] R. Benzi, A. Sutera, and A. Vulpiani, J. Phys. A 18, 2239 (1985).

[36] J. F. Lindner et al., Phys. Rev. Lett. 75, 3 (1995).

[37] J. F. Lindner et al., Phys. Rev. E 53, 2081 (1996).

[38] F. Marchesoni, L. Gammaitoni, and A. Bulsara, Phys. Rev. Lett. 76, 2609 (1996).

[39] J. J. Brey and A. Prados, Phys. Lett. A 216, 240 (1996).

[40] Z. Néda, Phys. Rev. E 51, 5315 (1995).

[41] Z. Néda, Phys. Lett. A 210, 125 (1996).

[42] P. M. Gade, R. Rai, and H. Singh, Phys. Rev. E 56, 2518 (1997).

[43] P. Jung, U. Behn, E. Pantazelou, and F. Moss, Phys. Rev. A 46, R1709 (1992).

[44] R. Glauber, J. Math. Phys. 4, 294 (1963).

[45] P. A. Rikvold, H. Tomita, S. Miyashita, and S. W. Sides, Phys. Rev. E 49, 5080 (1994).

[46] P. A. Rikvold and B. Gorman, in Annual Reviews of Computational Physics I, edited by D. Stauffer (World Scientific, Singapore, 1994), p. 149.

[47] P. A. Rikvold, M. A. Novotny, M. Kolesik, and H. L. Richards, in Dynamical Properties of Unconventional Magnetic Systems, edited by A. T. Skjeltorp and D. Sherrington (Kluwer, Dordrecht, 1997).

[48] S. W. Sides, R. A. Ramos, P. A. Rikvold, and M. A. Novotny, J. Appl. Phys. 79, 6482 (1996).

[49] S. W. Sides, R. A. Ramos, P. A. Rikvold, and M. A. Novotny, J. Appl. Phys. 81, 5597 (1997).

[50] S. W. Sides, P. A. Rikvold, and M. A. Novotny, In press J. Appl. Phys. Preprint cond-mat/9710244 (1988).

[51] S. W. Sides, P. A. Rikvold, and M. A. Novotny, In preparation (1998).

[52] M. Acharyya and B. K. Chakrabarti, in Annual Reviews of Computational Physics I, edited by D. Stauffer (World Scientific, Singapore, 1994), p. 107.

[53] M. Acharyya and B. K. Chakrabarti, Physica A 192, 471 (1992).

[54] M. Acharyya, B. K. Chakrabarti, and A. K. Sen, Physica A 186, 231 (1992).

[55] M. Acharyya and B. K. Chakrabarti, J. Magn. Magn. Mater. 136, L29 (1994).

[56] M. Acharyya, B. K. Chakrabarti, and R. Stinchcombe, J. Phys. A: Math. Gen. 27, 1533 (1994).

[57] W. Lo and R. A. Pelcovits, Phys. Rev. A 42, 7471 (1990).

[58] Z. Fan, Z. Jinxiu, and L. Xiao, Phys. Rev. E. 52, 1399 (1995).

[59] M. Acharyya, Phys. Rev. E 56, 1234 (1997).

[60] M. Acharyya, Physica A 235, 469 (1997).

[61] D. Dhar and P. B. Thomas, J. Phys. A: Math. Gen. 25, 4967 (1992).

[62] P. B. Thomas and D. Dhar, J. Phys. A: Math. Gen. 26, 3973 (1993).

[63] M. Acharyya and B. K. Chakrabarti, Phys. Rev. B 52, 6550 (1995).

[64] K. Binder and D. Heermann, Monte Carlo Simulation in Statistical Physics (Springer, Berlin, 1988).

[65] P. A. Martin, J. Stat. Phys. 16, 149 (1977).

[66] J. Lee, M. A. Novotny, and P. A. Rikvold, Phys. Rev. E 52, 356 (1995).

[67] The free-energy data shown in Fig. 1 were obtained by a multicanonical simulation of a $64 \times 64$ square-lattice Ising ferromagnet at $T=0.8 T_{c}$ as part of the research reported in Ref. 66. This figure is analogous to Fig. 1(a) of that paper, which shows the free energy for a $24 \times 24$ system at $H=0$.

[68] J. S. Langer, Ann. Phys. (N.Y.) 41, 108 (1967).

[69] J. S. Langer, Ann. Phys. (N.Y.) 54, 258 (1969).

[70] N. J. Günther, D. A. Nicole, and D. J. Wallace, J. Phys. A 13, 1755 (1980).

[71] C. C. A. Günther, P. A. Rikvold, and M. A. Novotny, Physica A 212, 194 (1994).

[72] R. A. Ramos, S. W. Sides, P. A. Rikvold, and M. A. Novotny, In preparation .

[73] D. Cox and H. Miller, The Theory of Stochastic Processes (Methuen, London, 1965).

[74] T. Zhou, F. Moss, and P. Jung, Phys. Rev. A 42, 3161 (1990).

[75] J. Gunton and M. Droz, Introduction to the Theory of Metastable and Unstable States (Springer, Berlin, 1983).

[76] I. Lifshitz, Sov. Phys. JETP 15, 939 (1962).

[77] S. Allen and J. Cahn, Acta Metall. 27, 1085 (1979).

[78] K. Sekimoto, Physica 135A, 328 (1986).

[79] V. Shneidman and P. Hänggi, Phys. Rev. E. 49, 641 (1994).

[80] P. Jung, Phys. Rep. 234, 175 (1993).

[81] L. Gammaitoni, F. Marchesoni, and S. Santucci, Phys. Rev. Lett. 74, 1052 (1995).

[82] W. Wernsdorfer et al., Phys. Rev. Lett. 78, 1791 (1997).

[83] W. H. Press, S. A. Teukolsky, W. T. Vetterling, and B. P. Flannery, Numerical Recipes in Fortran: The Art of Scientic 
Computing, 2nd ed. (Cambridge University Press, Boston, 1992).

[84] V. Shneidman, P. Jung, and P. Hänggi, Europhys. Lett. 26, 571 (1994).

[85] T. Zhou and F. Moss, Phys. Rev. A 41, 4255 (1990).

[86] S. W. Sides, Ph.D. thesis, In preparation (1998).

[87] M. Abramowitz and I. A. Stegun, Handbook of Mathematical Functions (National Bureau of Standards, Washington, D.C., 1970), p. 260.

[88] M. C. Mahato and S. R. Shenoy, Phys. Rev. E 50, 2503 (1994).

[89] M. C. Mahato and A. M. Jayannavar, Phys. Rev. E 55, 6266 (1997).

[90] M. Acharyya, Preprint cond-mat/9712309 (1998).

\begin{tabular}{|c|c|c|c|c|c|}
\hline \multicolumn{2}{|c|}{ Parameters } & \multicolumn{2}{|c|}{ Constants (theory) } & \multicolumn{2}{|c|}{ Constants (simulation) } \\
\hline$H_{0}$ & $0.1 J$ & $\Xi_{0}(T)$ & $0.506192 \mathrm{~J}$ & $\Omega$ & 3.15255 \\
\hline$L$ & 64 & $K$ & 3 (exact) & $\nu$ & $(0.465 \pm 0.014) J^{-1} \mathrm{MCSS}^{-1}$ \\
\hline$T$ & $0.8 T_{c}$ & & & $\langle\tau\rangle$ & $2058 \mathrm{MCSS}$ \\
\hline & & & & $r$ & 0.672 \\
\hline
\end{tabular}

TABLE I. Parameters and constants used in this work. The values of the parameters $H_{0}, L$, and $T$ have been selected such that switching occurs via the single-droplet mechanism, while the maximum nucleation rate is not too low to obtain reasonable simulation statistics. The constants $\Xi_{0}(T)$ and $K$ are calculated from droplet theory [68 71] for two-dimensional Ising systems. The constants $\langle\tau\rangle$ and $r$ are measured from field-reversal MC simulations with the Glauber dynamic (using the parameters listed above). The constants $\Omega \sqrt[71]{71}$ and $\nu \sqrt[72]{72}$ have been measured in other work (for clarity, we do not explicitly show the temperature dependence of these quantities in the table or elsewhere in the paper). The value for $H_{\mathrm{DSP}}$ is taken from Fig. 11 of Ref. 66 . 


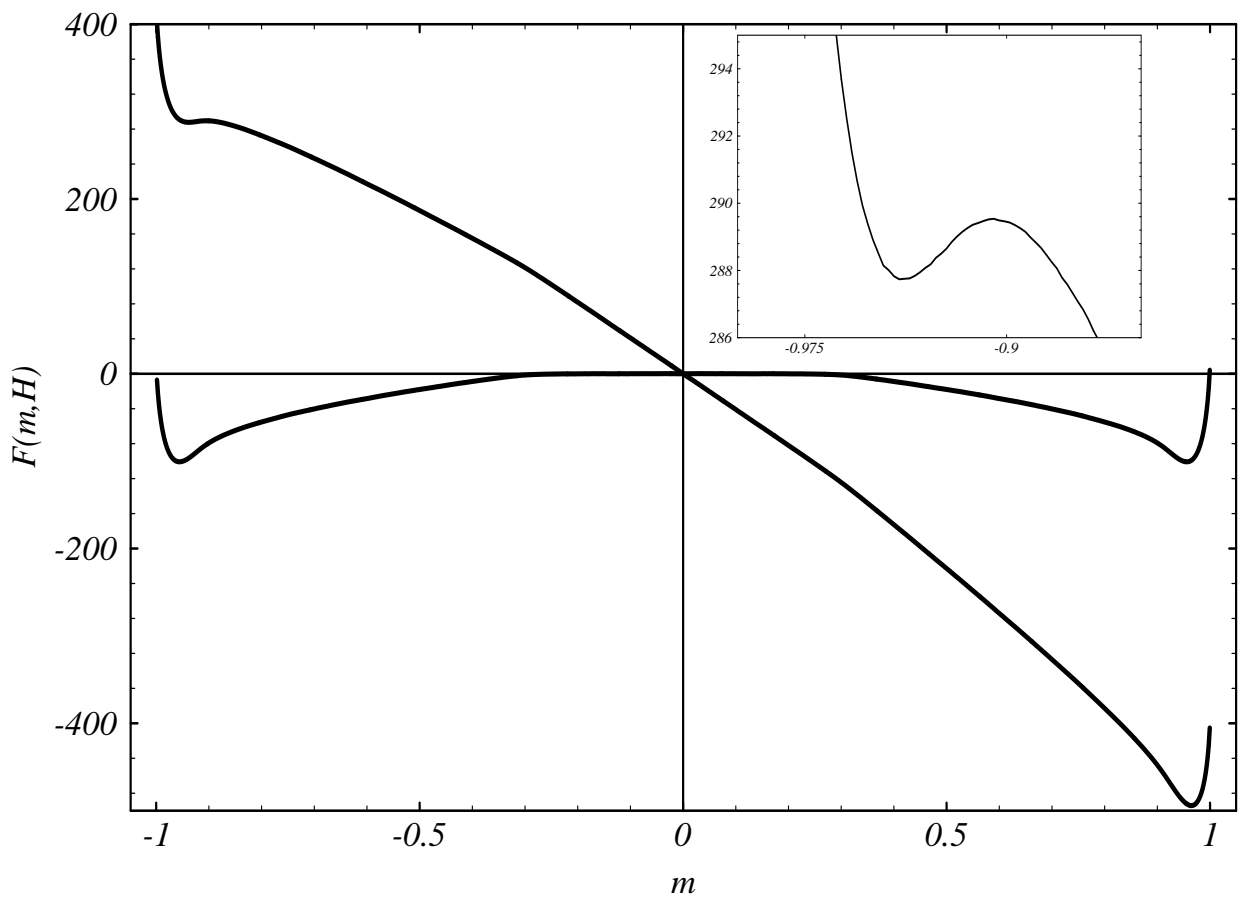

FIG. 1. The free energy, $F(m, H, T)$, shown vs. magnetization $m$ for the nearest-neighbor Ising ferromagnet on a $64 \times 64$ square lattice at $T=0.8 T_{c}$. Data are shown for $H=0.0 \mathrm{~J}$ and $0.1 \mathrm{~J}$. The data was obtained from a study in which a multicanonical MC algorithm was used to find $F(m, 0, T)$ [66. The inset shows an expanded view of the portion of the free energy curve near the metastable state for $H=0.1 J$. The barrier height is on the order of $1 k_{\mathrm{B}} T$. 

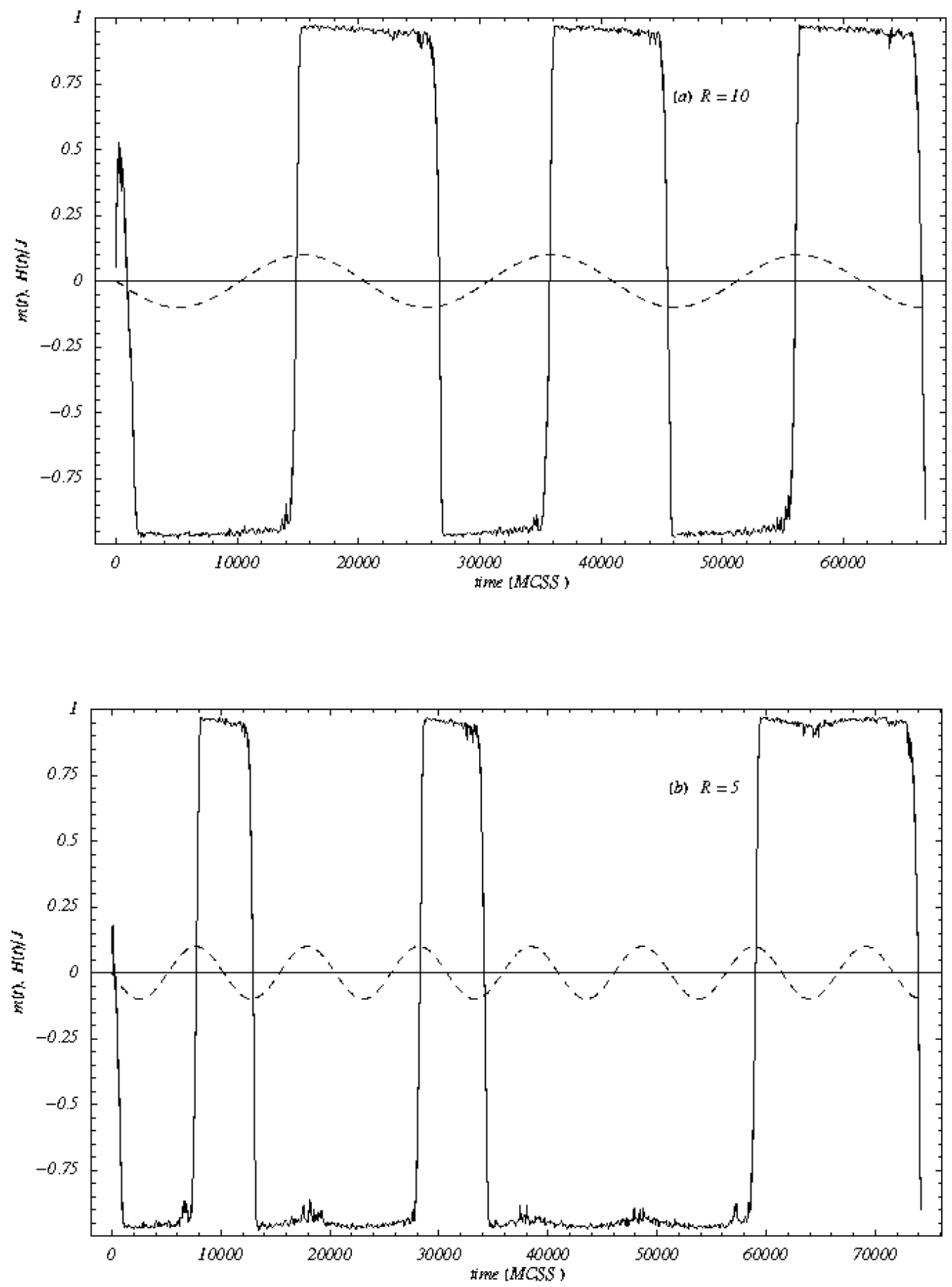


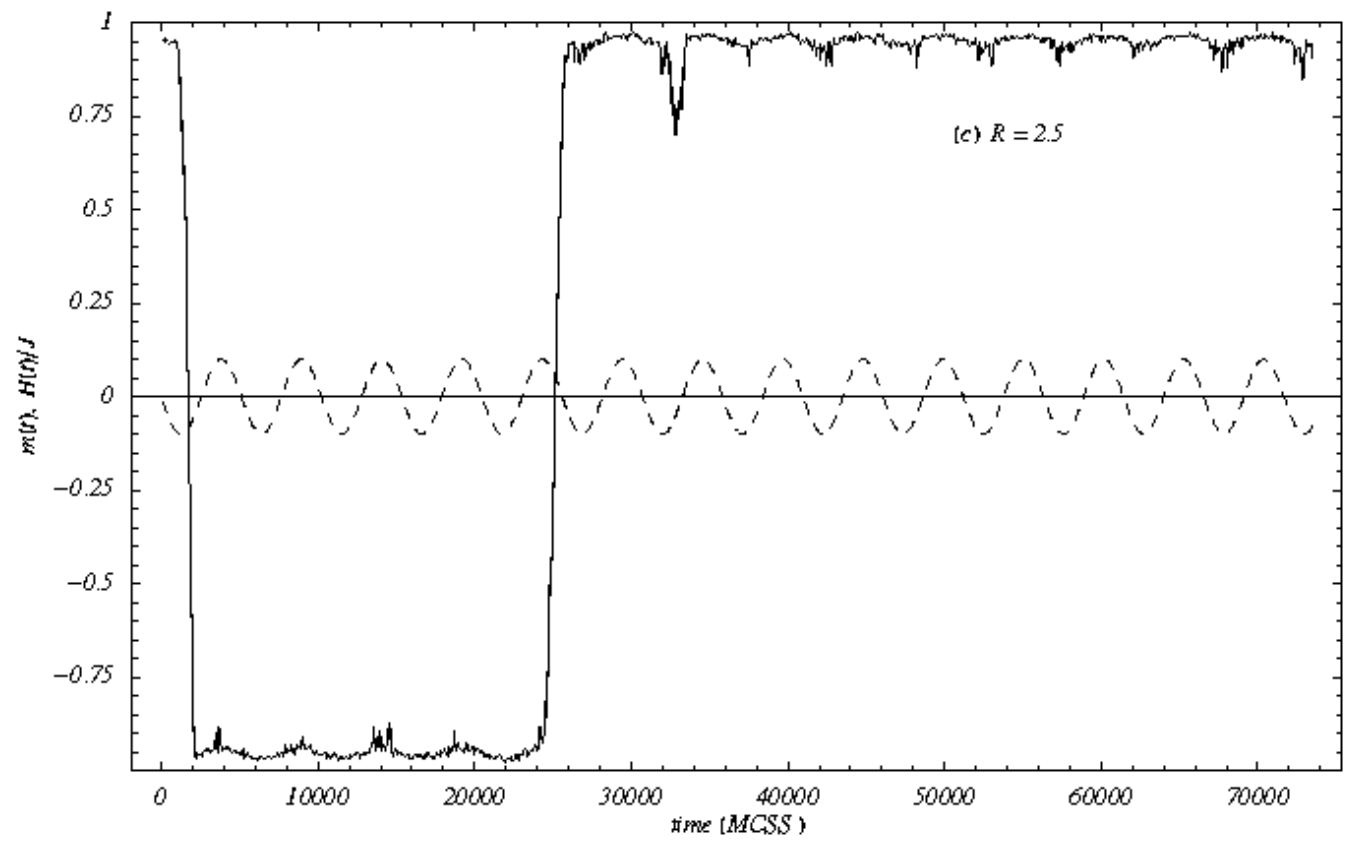

FIG. 2. Short initial segments of the magnetization time series $m(t)$ (solid line) and the external field $H(t)$ (dashed line) vs. time $t$ in the SD region. for $T=0.8 T_{r}, d=2 . L=64$. and $H_{n}=0.1 J$. The total length of the time series is approximately $16.9 \times 1$

. The time series are shown $\mathrm{f}$

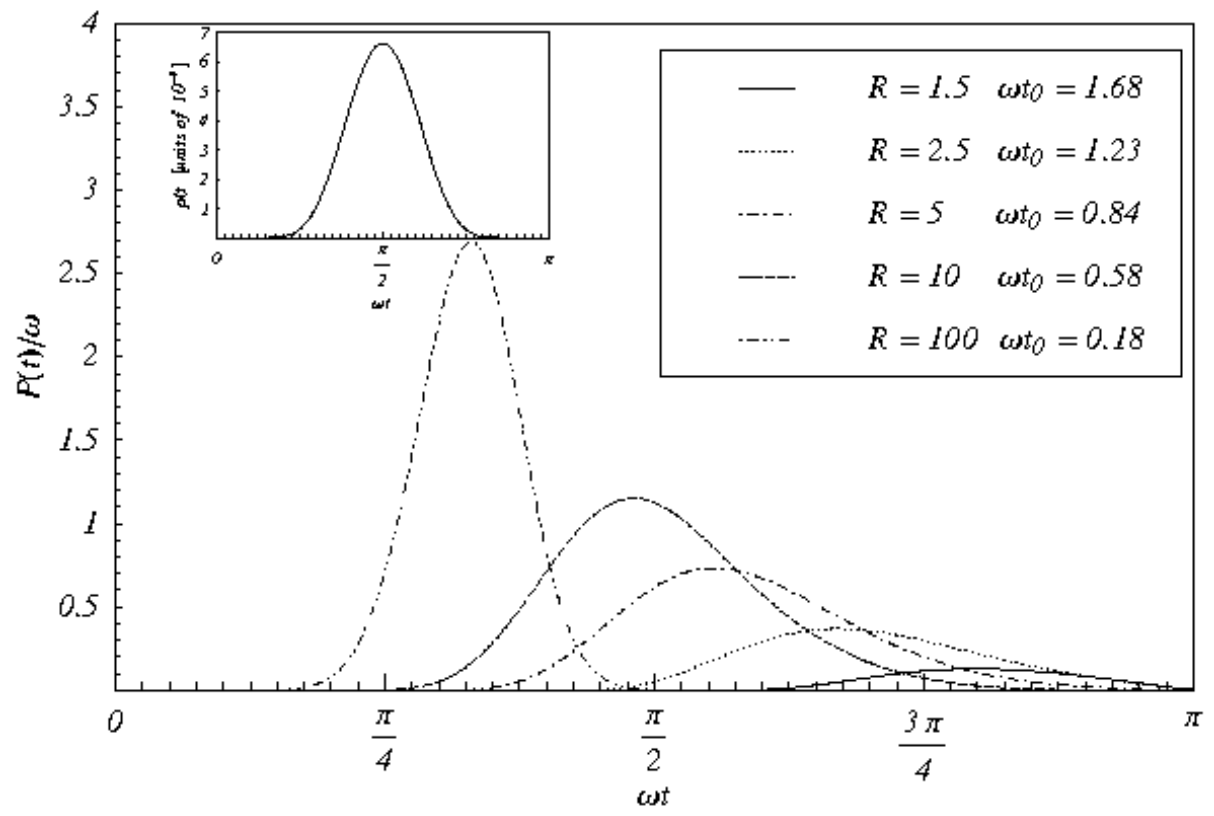

FIG. 3. The switching probability density, $P(t) / \omega$ vs. $\omega t$, for five values of the period of the external field, $R=1.5, R=2.5$, $R=5, R=10$, and $R=100$. The plots are obtained from a numerical evaluation of Eq. (4.14). The inset shows the decay rate, $\rho(t)$ vs. $\omega t$. The time $t_{0}$ is the earliest time during a period, for which $P(t)$ is non-zero. Even though the decay rate always has a maximum when the phase equals $\pi / 2$, the value of the phase for which $P(t) / \omega$ is maximum depends on the frequency. 

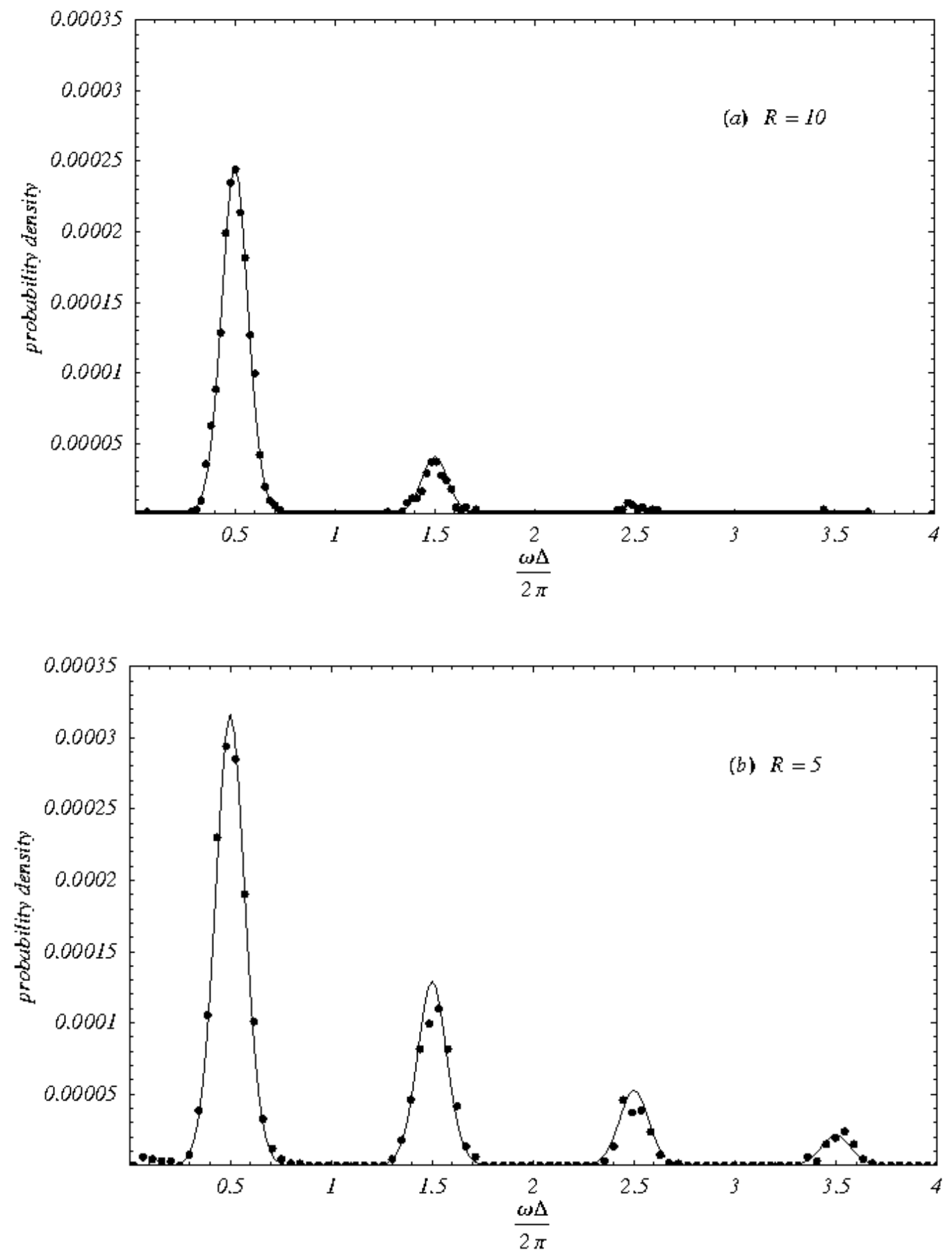


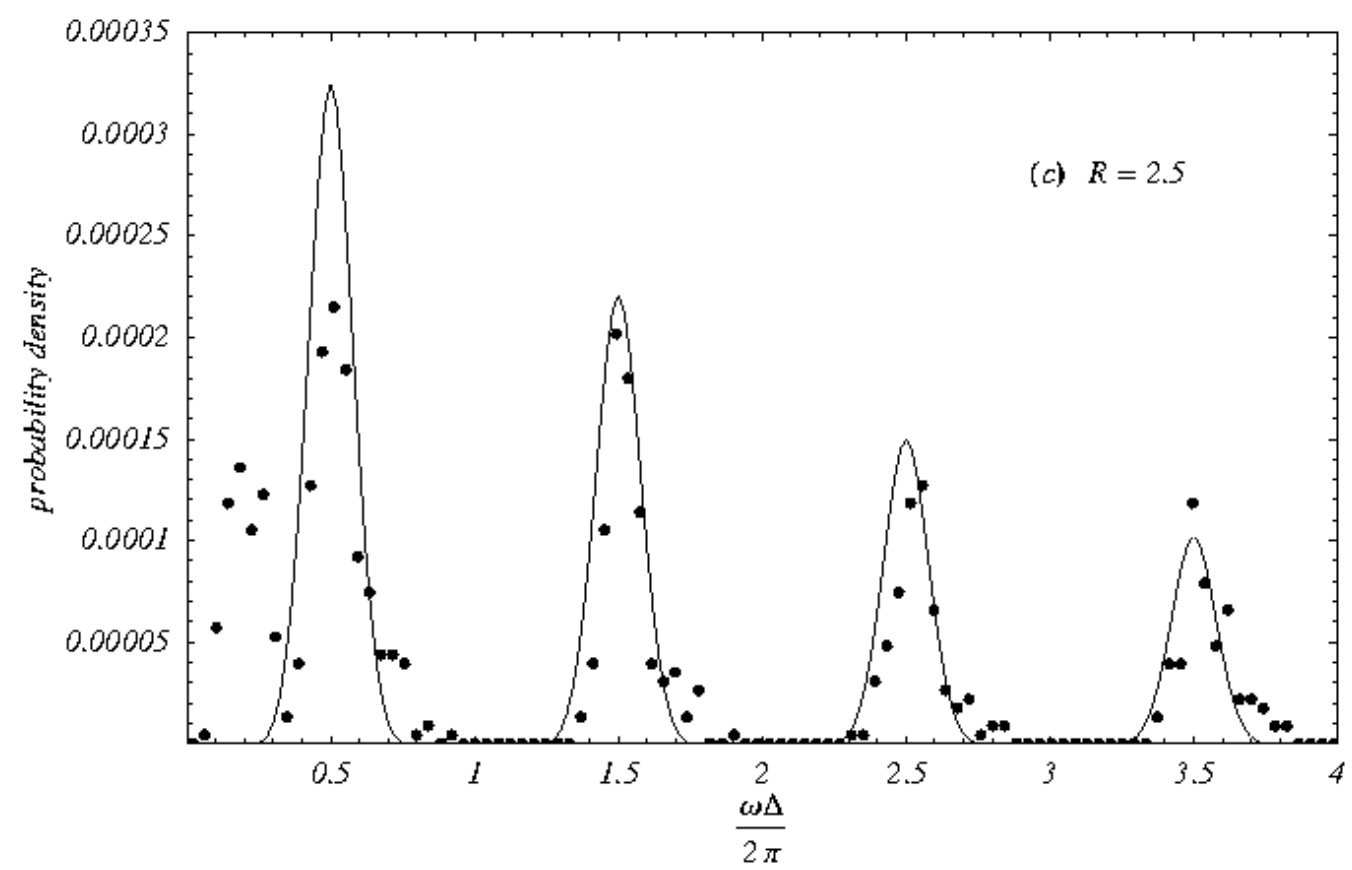

FIG. 4. Residence-time distributions (RTDs). The time axis is scaled by the period $2 \pi / \omega$ of the external field for each value of $R$, so that the peaks are centered around odd half-integer multiples. The RTDs are shown for (a) $R=10$ (150 bins, 1239 events), (b) $R=5$ (250 bins, 1439 events), and (c) $R=2.5$ (500 bins, 1089 events). The filled circles are obtained from the MC simulations. The solid curves represent the theoretical calculation presented in Appendix A.

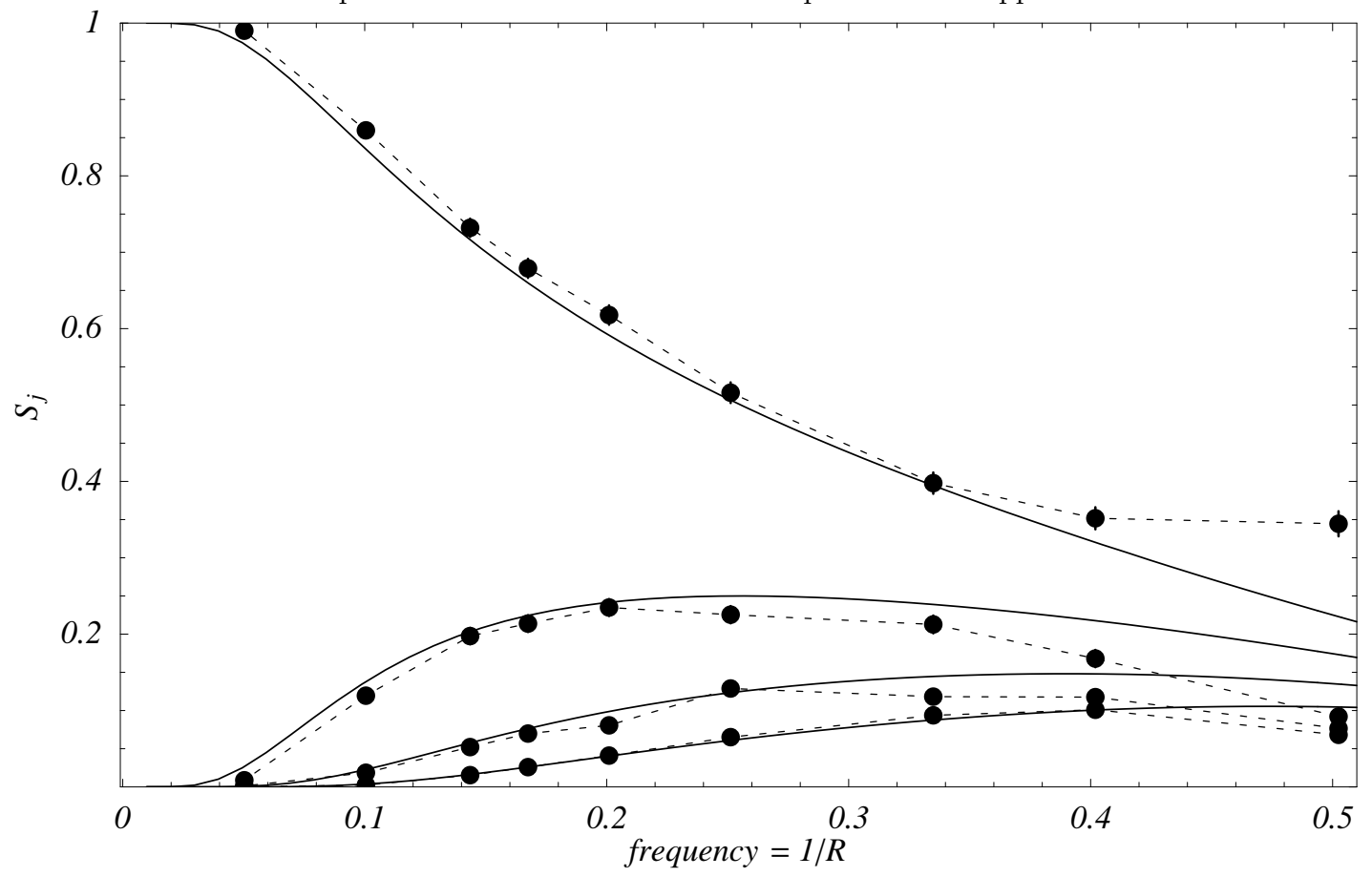

FIG. 5. Peak strengths in the RTDs vs. scaled frequency $1 / R$. The different curves, from top to bottom, correspond to $S_{1}$, $S_{2}, S_{3}$, and $S_{4}$. The statistical errors are everywhere on the order of the symbol size or less. The solid curves, obtained from Eq. (5.4), result from the same parameter-free calculation as the RTDs. 


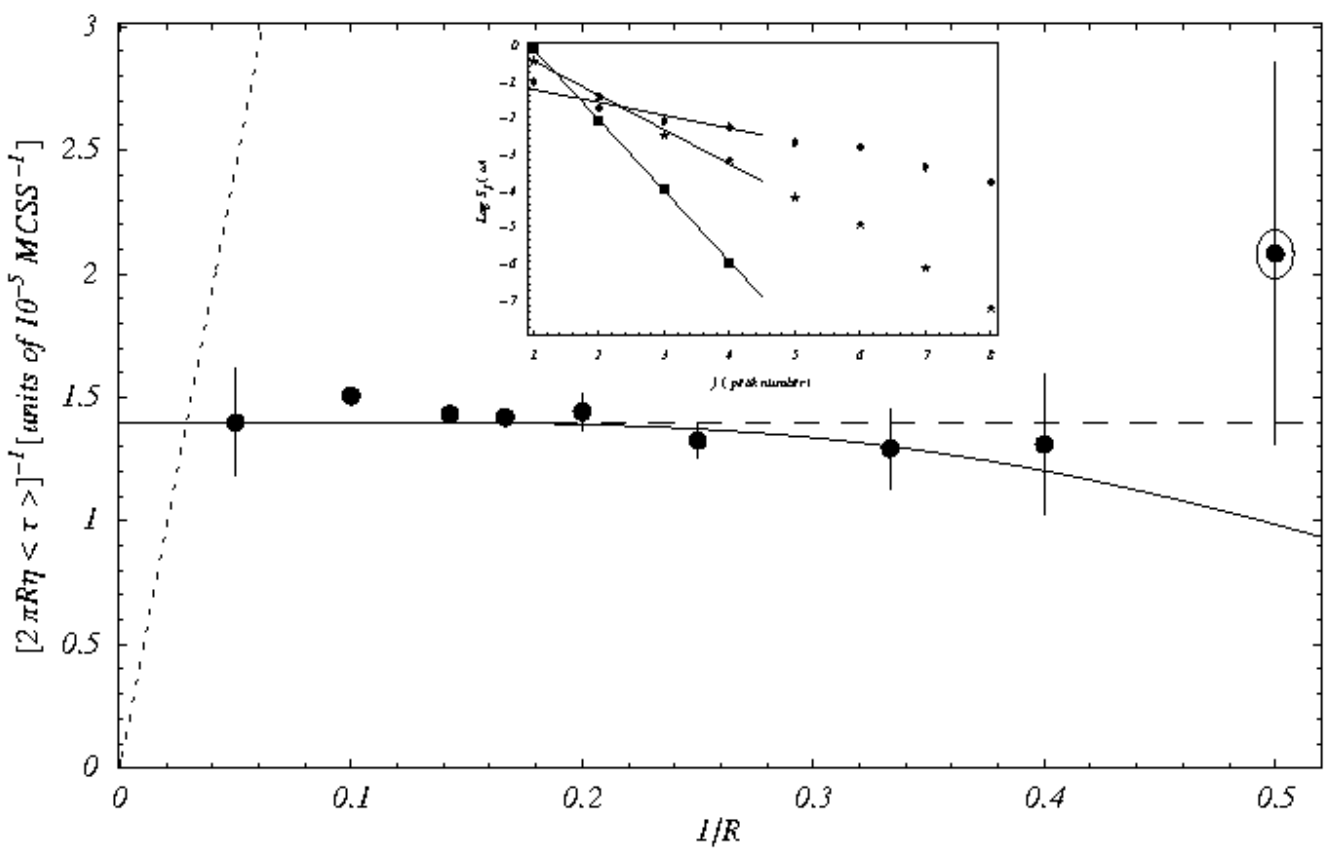

FIG. 6. The inverse characteristic time of the RTDs, $[2 \pi R \eta\langle\tau\rangle]^{-1}$, vs. the scaled frequency, $1 / R$. The inverse characteristic time is given in units of $10^{-5} \mathrm{MCSS}^{-1}$. The solid dots are calculated from MC data for the peak strengths of the RTDs. The inset shows examples of $\ln S_{j}$ vs. the peak number $j$, along with their linear least-squares fit lines. The three frequencies shown in the inset are $1 / R=0.4,0.2$, and 0.1 , denoted by diamonds, stars, and squares respectively. The error bars on the data are calculated from the standard deviation in the slopes of the fitted lines. The solid curve is obtained from the full numerical calculation of Eq. (5.7). The horizontal long-dashed line results from a low-frequency approximation obtained by setting $t_{\mathrm{g}}=0$. This horizontal line is located at a value of $[2 \pi R \eta\langle\tau\rangle]^{-1}=1.4 \times 10^{-5} \mathrm{MCSS}^{-1}$ obtained by numerical integration of Eq. (5.11). The short-dashed line represents the frequency, $\langle\tau\rangle / R$, which has been scaled to have the units of MCSS ${ }^{-1}$. The open oval around the point for $1 / R=0.5$ is a reminder of the poor statistics and large systematic error in the RTD for this frequency. 


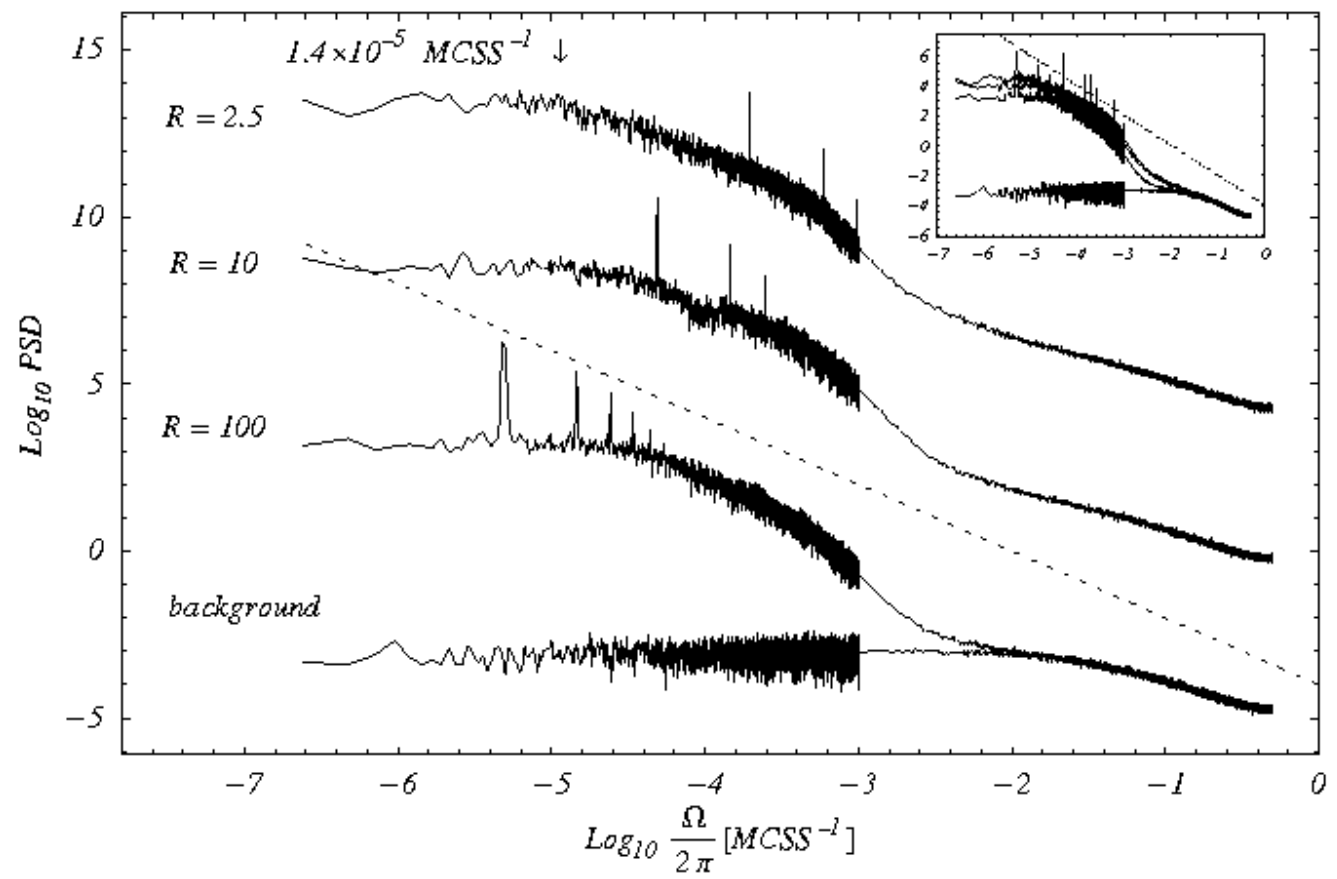

FIG. 7. Power spectral densities (PSDs). Spectra are shown for three different frequencies of the external field, and are plotted with an arbitrary offset for clarity. The inset shows the same spectra without the offset to illustrate how all three PSDs fall onto the thermal noise background at high frequencies. In addition to the change in the amount of smoothing, the right-hand section of each spectrum contains only one data point out of every 25 to facilitate plotting. The magnetization is sampled every 1.0 MCSS, so the Nyquist frequency is $0.5 \mathrm{MCSS}^{-1}$. The lowest frequency that can be resolved is $2.38 \times 10^{-7} \mathrm{MCSS}^{-1}$. The dashed line with slope -2 is a guide to the eye. The arrow indicates a frequency of $1.4 \times 10^{-5} \mathrm{MCSS}^{-1}$ in the PSD. This frequency value is the half-width of the spectrum predicted in Sec. $\mathrm{VO}$ for low frequencies of the external field. 


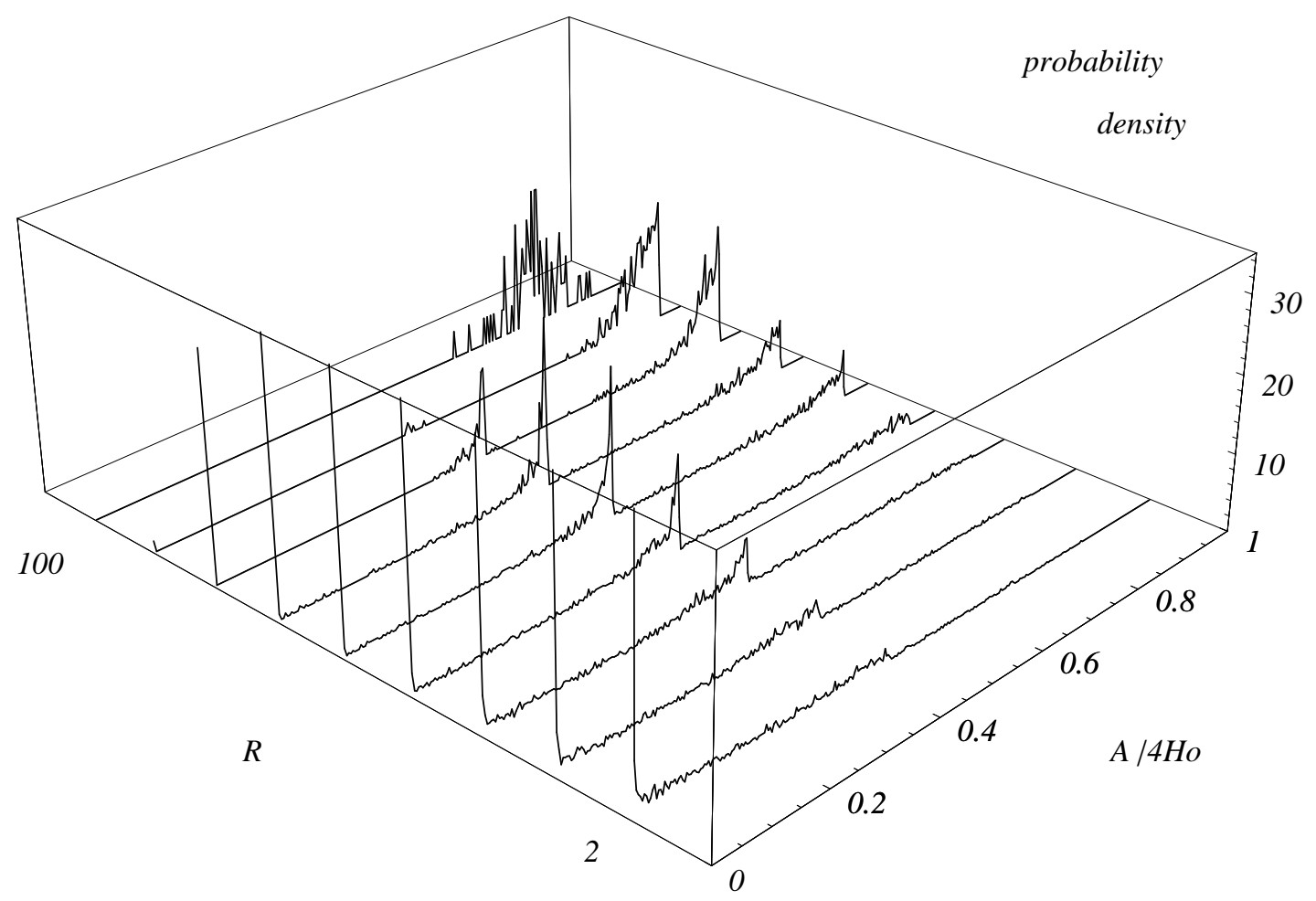

FIG. 8. Probability densities for the hysteresis-loop area, $A=-\oint m(H) d H$. The values of $R$ shown here are $R=2,2.5,3$, $4,5,6,10,20$, and 100 .
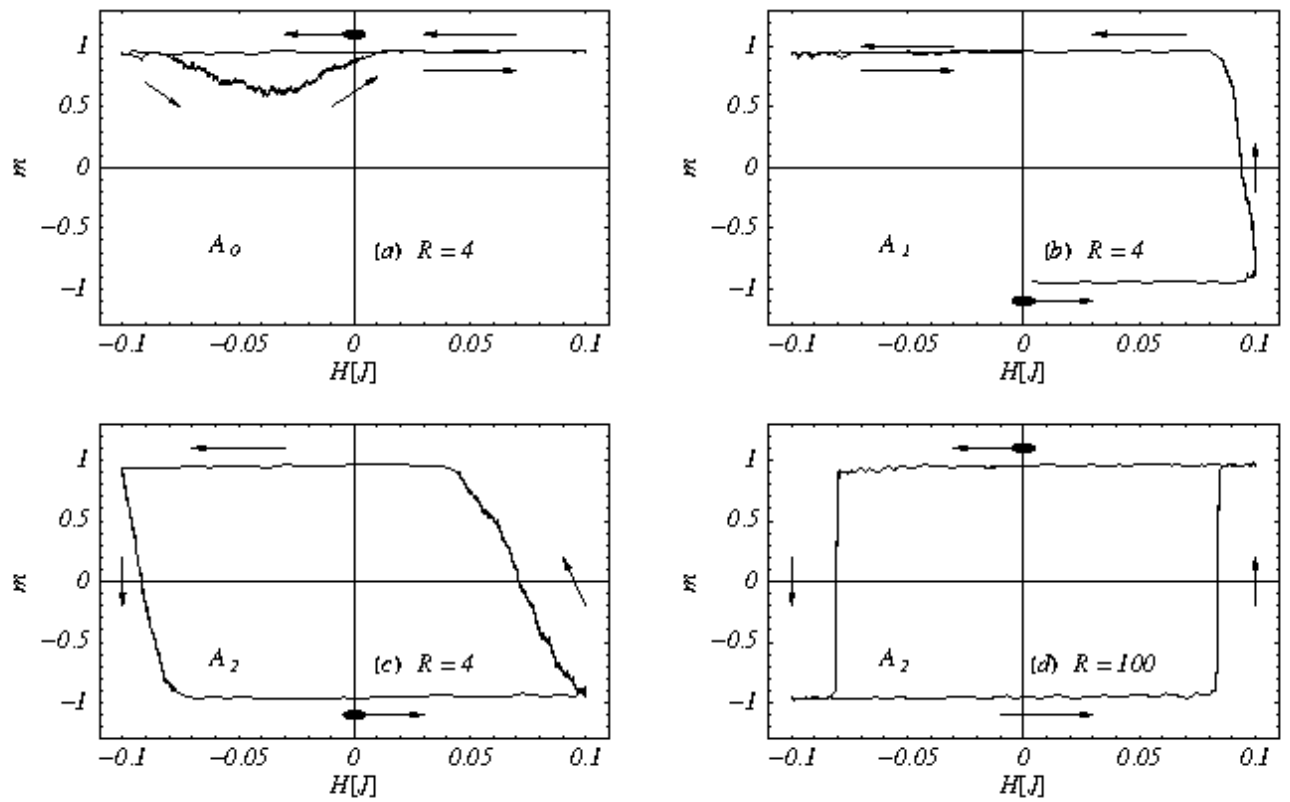

FIG. 9. Representative hysteresis loops corresponding to different numbers of switching events during one period of the applied field. Each loop in panels (a)-(c) consists of data from a single period in a time series with $R=4$. For $A_{0}$, no magnetization reversal occurs. This example shows a "spike" in the magnetization during one half of the period. For $A_{1}$, one magnetization reversal occurs during the period. For $A_{2}$ in panels (c) and (d), two magnetization reversals occur. Panel (d) shows a hysteresis loop of type $A_{2}$ from a very low-frequency time series with $R=100$. Note: the arrow tails with filled dots indicate the start of the field cycle. 

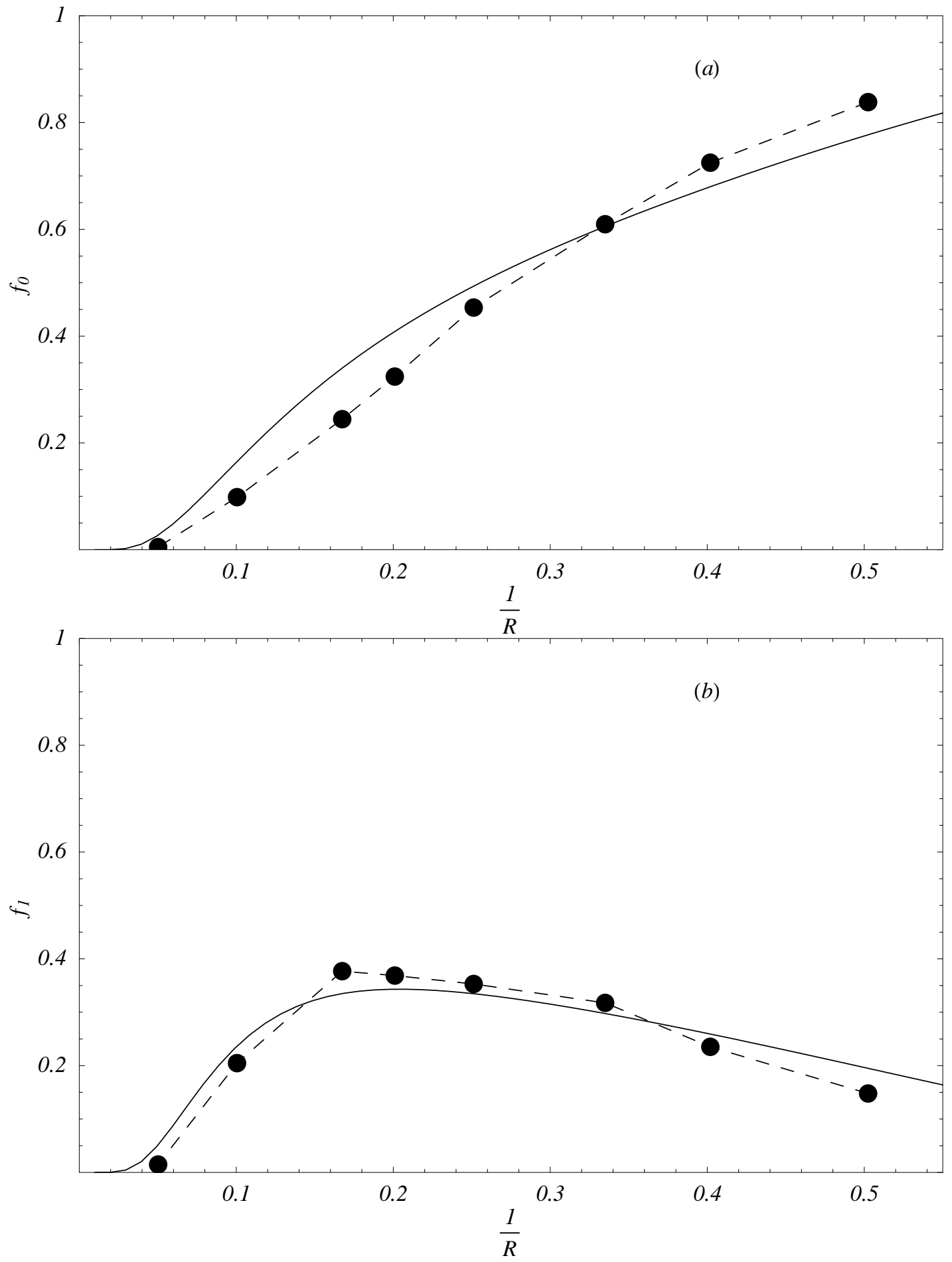

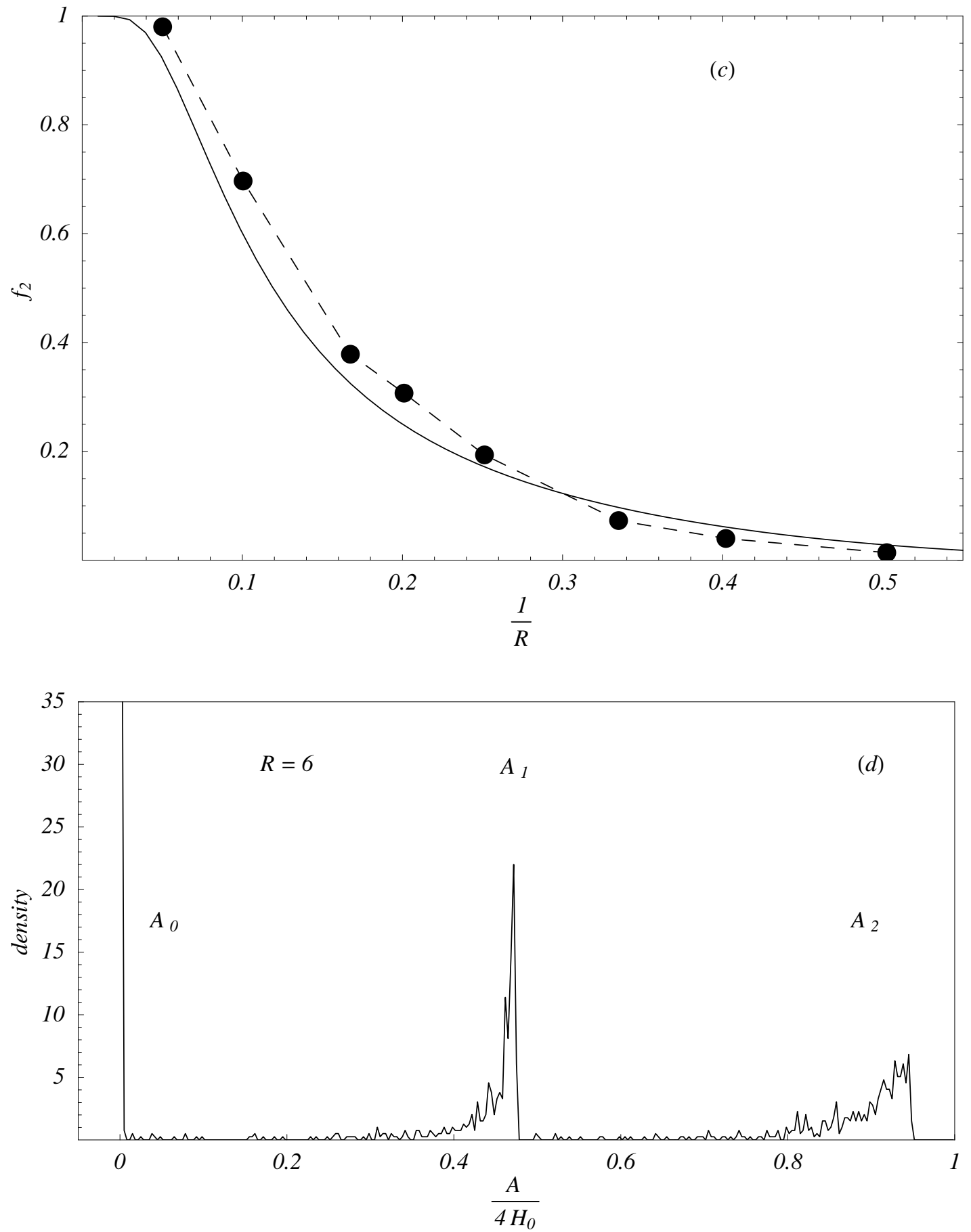

FIG. 10. Frequency dependence of the fraction of events contained in the peaks (a) $A_{0}$, (b) $A_{1}$, and (c) $A_{2}$. The solid dots are measured directly from the data in the loop-area distributions, and the dashed lines are guides to the eye. The solid curve in each plot is calculated using Eqs. (7.5a-c) with $P_{\text {not }}(\omega)$ obtained from a numerical evaluation of Eq. (4.18). (d) The loop-area distribution for $R=6$ is shown to illustrate the peak sizes in the trimodal distribution. 


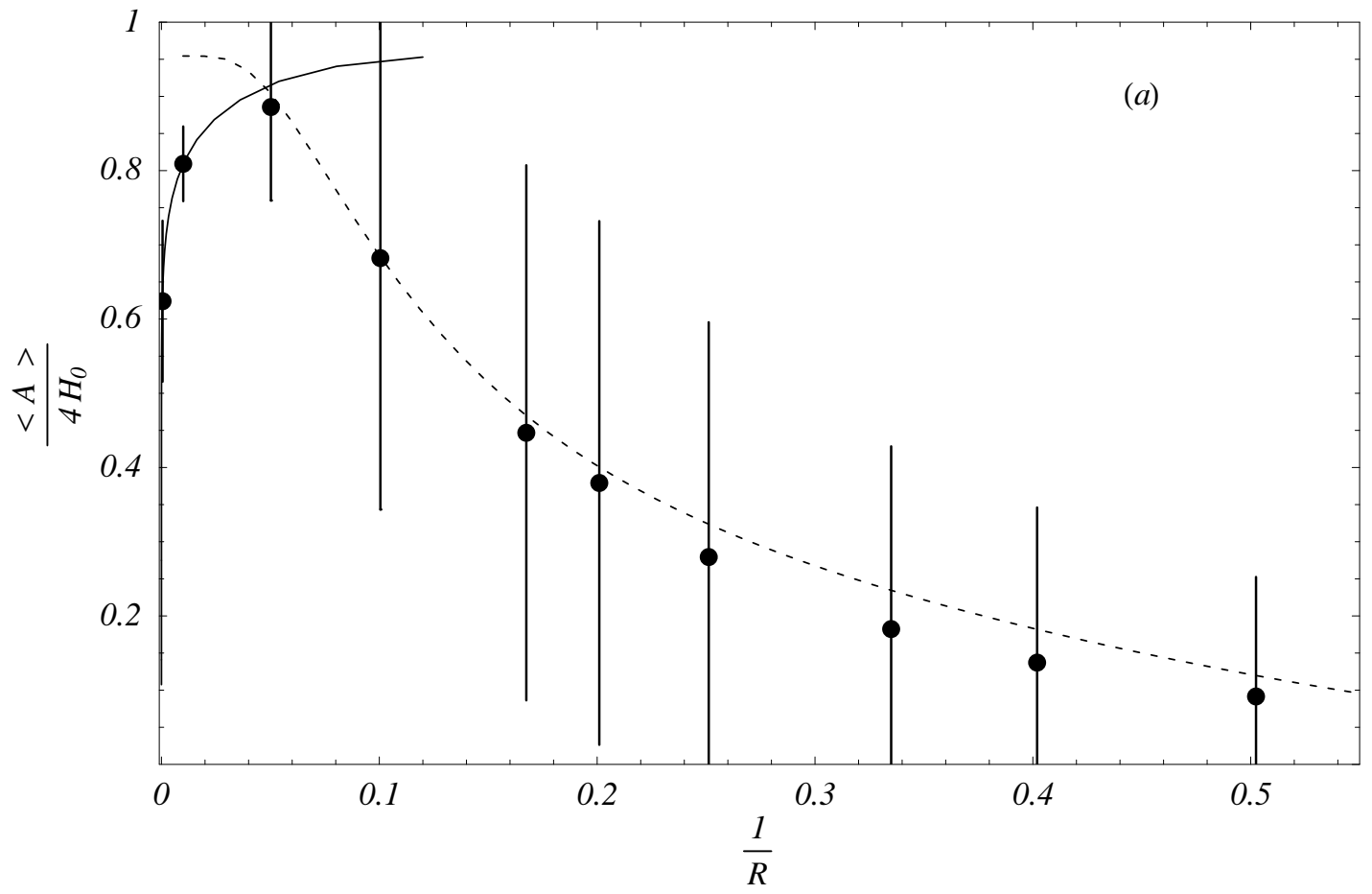




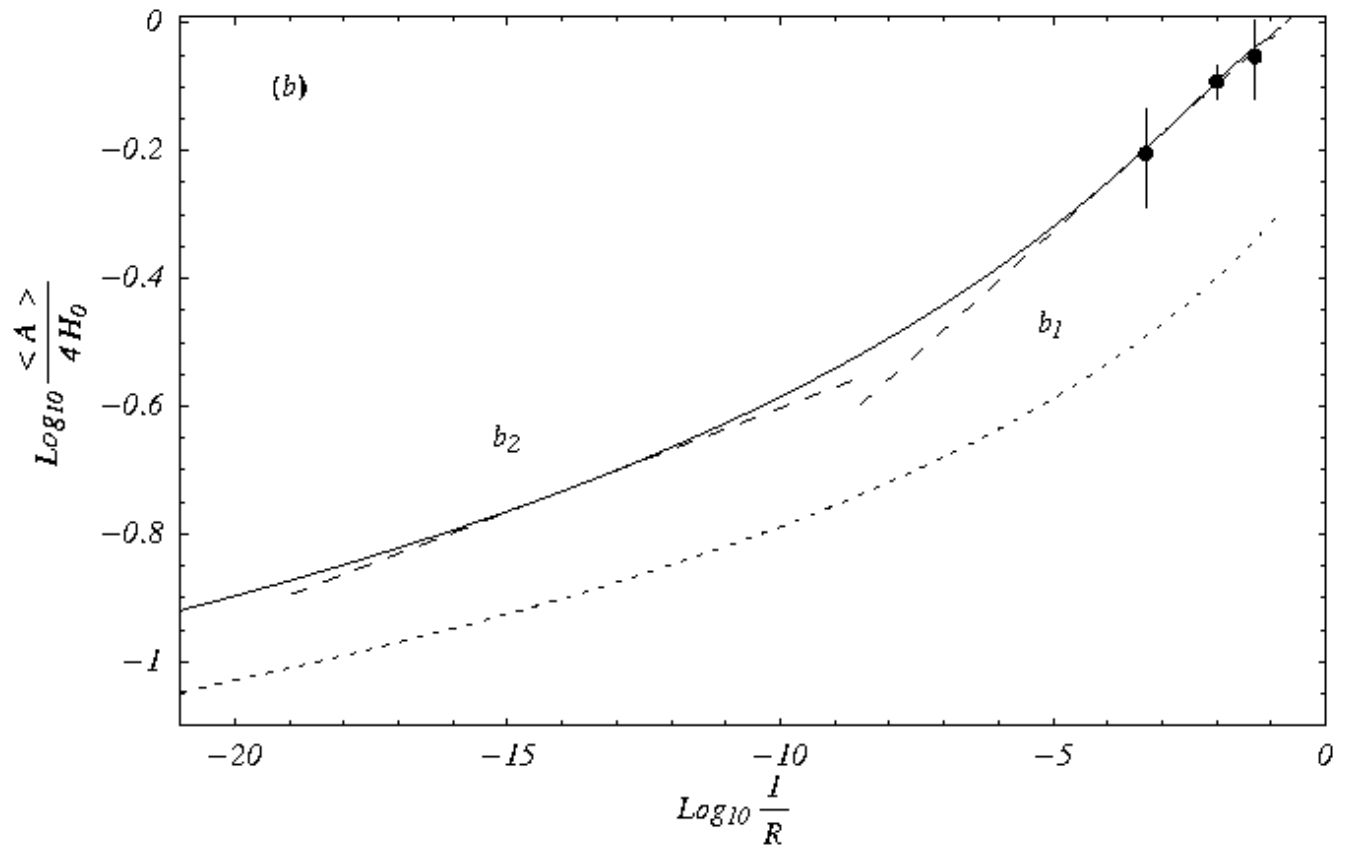

FIG. 11. (a). Mean and standard deviation of the loop-area distributions shown vs. the scaled frequency, $1 / R$. The solid dots are the means of the distributions shown in Fig. 8. The vertical bars are not error bars, but give the standard deviations of those distributions. The large standard deviations for $1 / R \gtrsim 0.1$ indicate the trimodal distribution of the loop areas, typical of the SD region. For the points at the two lowest frequencies the magnetization switches nearly every half-period, giving a loop-area distribution which is close to unimodal. The solid and dotted curves come from two separate theoretical calculations. The solid curve results from numerical solution of an analytic expression for the switching field that assumes switching occurs during every half-period. The dotted curve comes from a calculation which uses the same values of $P_{\text {not }}(\omega)$ used for the theoretical curve in Fig. 10. (b). Log-log plot for the very lowest frequencies in panel (a). The solid curve is the same as the solid curve in panel (a). The long-dashed line segments represent linear least-squares fits to different portions of the numerical solution data, each covering almost four decades in frequency. The data that yield the effective exponent $b_{1}=0.077$, are centered around $\log _{10}(1 / R)=-3.35$; those that yield $b_{2}=0.033$ are centered around $\log _{10}(1 / R)=-13.78$. The solid dots are MC simulation data. The short-dashed curve represents Eq. (7.16) with $d=2$ and $C=0.101 J^{-1}$ MCSS. 


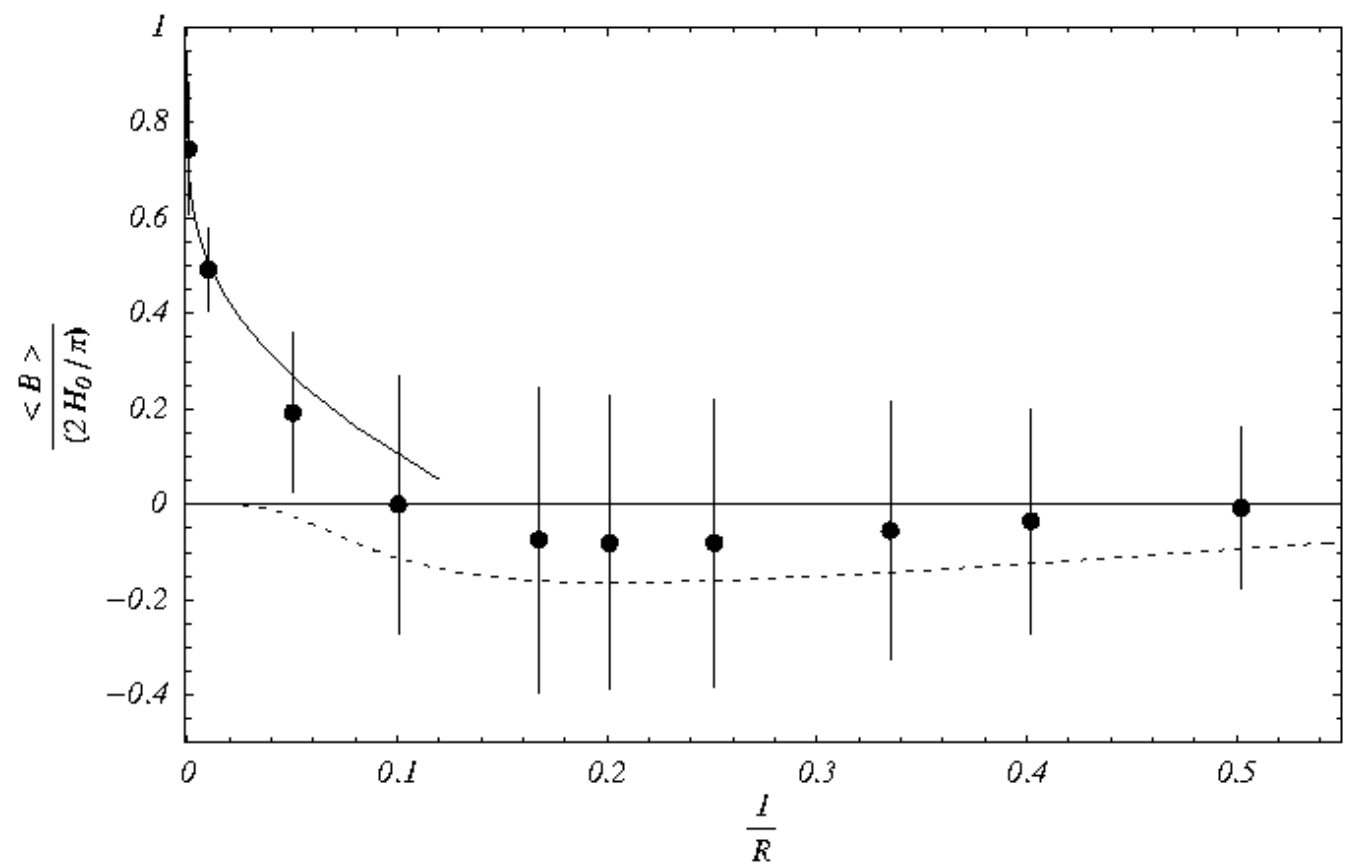

FIG. 12. Mean and standard deviation of the correlation, $B$ shown vs. the scaled frequency, $1 / R$. The solid dots are the means of the correlation distributions. The vertical bars are not error bars, but give the standard deviations of those distributions. The solid and dotted curves are obtained from similar calculations as those for the loop areas in Fig. 11 .

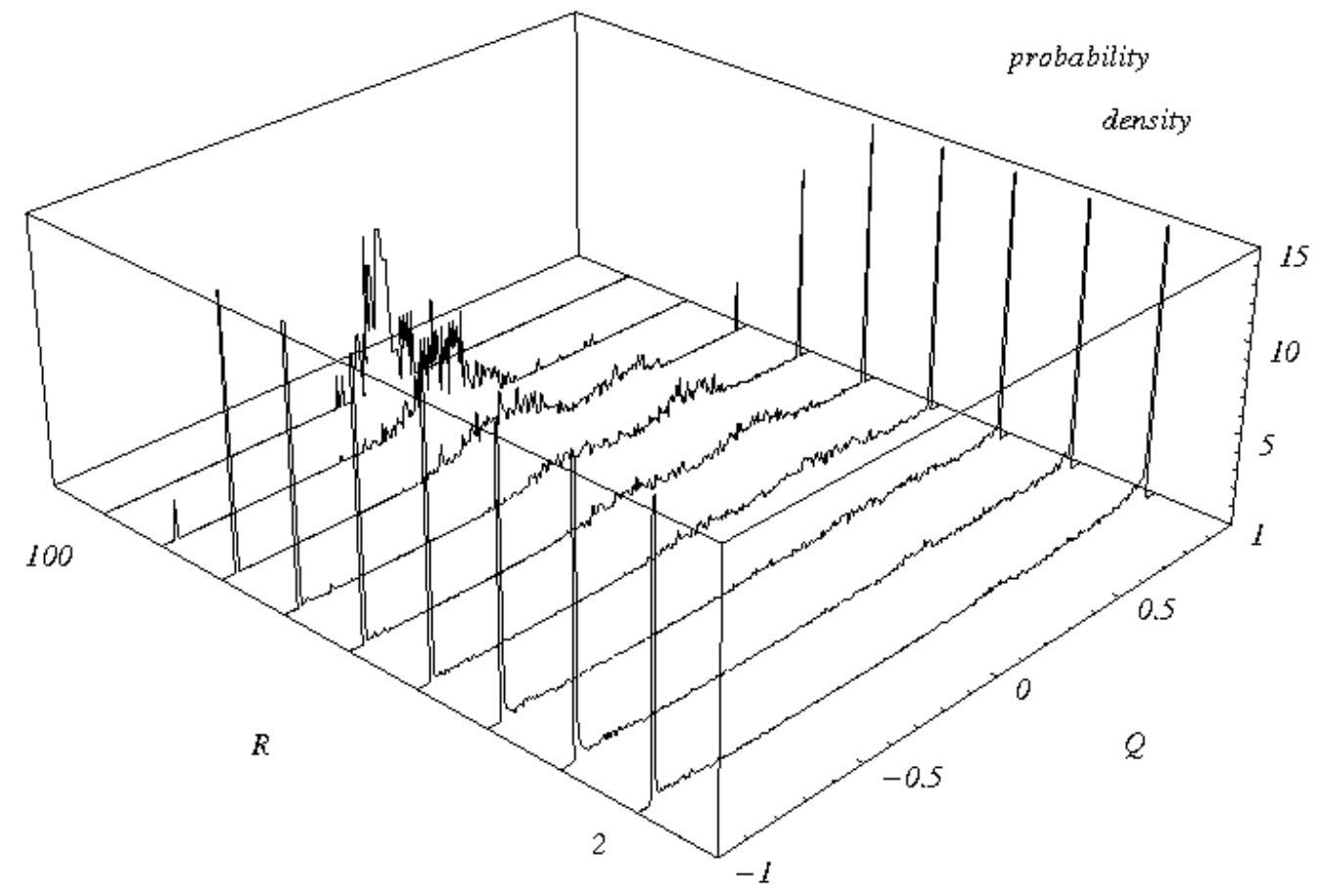

FIG. 13. Probability densities for the period-averaged magnetization, $Q$. The values of $R$ shown are $R=2,2.5,3,4,5,6$, 10,20 , and 100 . 
(a)

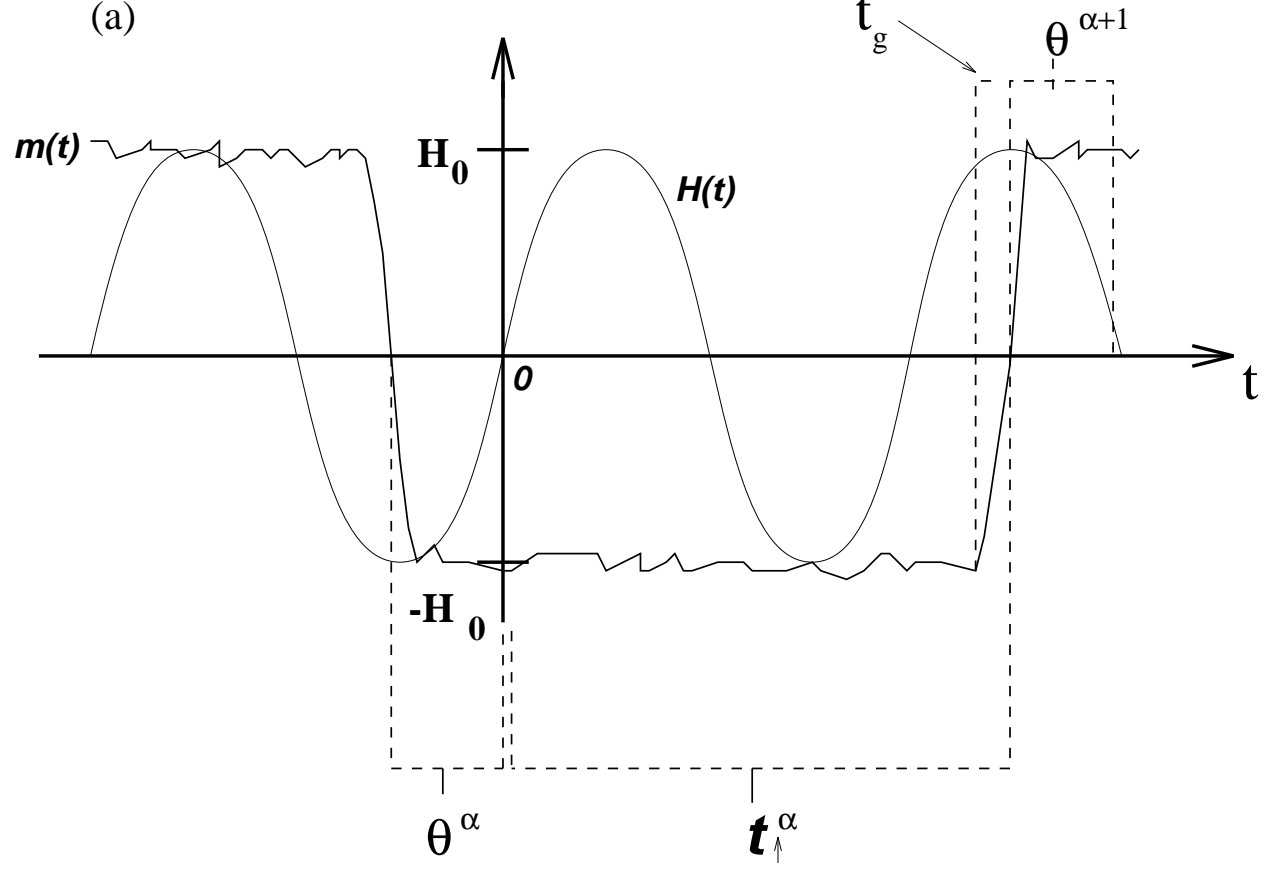

(b)

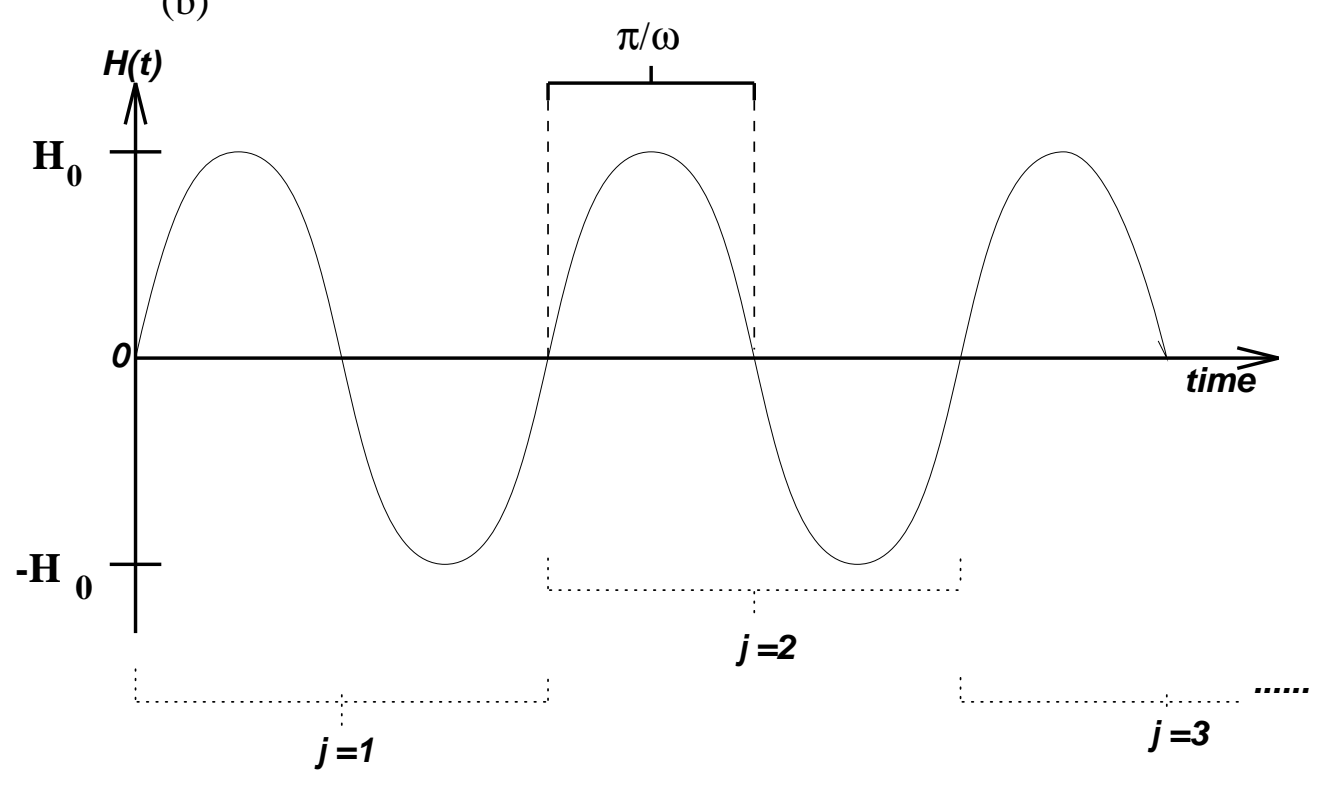

FIG. 14. Schematic diagrams for calculation of the RTDs. 